\title{
Ulam-Hyers-Mittag-Leffler stability for tripled system of weighted fractional operator with TIME delay
}

\author{
Mohammed A. Almalahi ${ }^{1,2}$, Satish K. Panchal ${ }^{1}$, Fahd Jarad ${ }^{3,4^{*}}$ (ID) and Thabet Abdeljawad ${ }^{4,5,6^{*}}$ (D)
}

"Correspondence:

fahd@cankaya.edu.tr;

tabdeljawad@psu.edu.sa

${ }^{3}$ Department of Mathematics,

Çankaya University, 06790

Etimesgut, Ankara, Turkey

${ }^{5}$ Department of Mathematics and

General Sciences, Prince Sultan

University, Riyadh, Saudi Arabia

Full list of author information is

available at the end of the article

\section{Springer}

\begin{abstract}
This study is aimed to investigate the sufficient conditions of the existence of unique solutions and the Ulam-Hyers-Mittag-Leffler (UHML) stability for a tripled system of weighted generalized Caputo fractional derivatives investigated by Jarad et al. (Fractals 28:2040011 2020) in the frame of Chebyshev and Bielecki norms with time delay. The acquired results are obtained by using Banach fixed point theorems and the Picard operator $(\mathrm{PO})$ method. Finally, a pertinent example of the results obtained is demonstrated.
\end{abstract}

MSC: $34 \mathrm{~A} 08 ; 34 \mathrm{~B} 15 ; 34 \mathrm{~A} 12 ; 47 \mathrm{H} 10$

Keywords: Fractional differential equation; Weighted Caputo operator; Fixed point theorem

\section{Introduction}

The topic of fractional differential equations (FDEs) has attracted the interest of researchers from various disciplines thanks to it being considered a useful gizmo in modeling the dynamics of various physical systems and their applications in many fields of applied sciences, engineering and technical sciences, etc. For further details, we refer the readers to [2-5].

There are various definitions of fractional calculus (FC) that developed the (FDEs) in modeling and describe the memory accurately. Among these famous operators are Riemann-Liouville, Riemann, Grunwald-Letnikov, Caputo, Hilfer and Hadamard which are the foremost used. For more details, we refer the readers to [1, 6-17]. There is a prominent and noticeable interest within the investigation of qualitative characteristics of solutions (existence, uniqueness, stability) of (FDEs). For applications and up-to-date work, we refer the readers to [18-26].

On the other hand, the study of coupled systems involving (FDEs) is additionally important as intrinsically systems occur in various problems of applied nature. For a few theoretical works on coupled systems of (FDEs), we refer to a series of papers [27-31].

(c) The Author(s) 2021. This article is licensed under a Creative Commons Attribution 4.0 International License, which permits use, sharing, adaptation, distribution and reproduction in any medium or format, as long as you give appropriate credit to the original author(s) and the source, provide a link to the Creative Commons licence, and indicate if changes were made. The images or other third party material in this article are included in the article's Creative Commons licence, unless indicated otherwise in a credit line to the material. If material is not included in the article's Creative Commons licence and your intended use is not permitted by statutory regulation or exceeds the permitted use, you will need to obtain permission directly from the copyright holder. To view a copy of this licence, visit http://creativecommons.org/licenses/by/4.0/ 
The topic of stability of systems is one among the foremost important qualitative characteristics of a solution. But as far as we know, this is often the primary work with regard to a tripled system of weighted fractional differential equations with time delay.

Tripled fractional boundary systems may be a generalization of coupled fractional systems as they are governed by three associated differential equations with three conditions $[32,33]$.

Recently, Matar et al. [34], by means of some fixed point theorems like Banach and Krasnoselskii, studied the existence and uniqueness of solutions of the tripled system

$$
{ }^{C} \mathcal{D}_{0^{+}}^{\kappa_{i}} \varsigma_{i}(\varkappa)=f_{i}(\varkappa, \varsigma(\varkappa)), \quad \kappa_{i} \in(1,2]
$$

with cyclic permutation boundary conditions

$$
\zeta_{i}^{(j)}(0)=a_{k, j} \zeta_{\sigma(k)}^{(j)}(T), \quad i=1,2,3, j=0,1
$$

where ${ }^{C} \mathcal{D}_{0^{+}}^{\kappa_{i}}$ denotes the Caputo fractional derivative (CFD) of order $\kappa_{i}, i=1,2,3, f_{i}$ : $[0, T] \times \mathbb{R}^{3} \rightarrow \mathbb{R}$ are continuous functions, $\sigma=(1,2,3)$ is a cycle permutation.

Wang and Zhang [35], by means of the Banach fixed point theorem and the Picard operator method, studied existence, uniqueness, and UHML stability results with respect to Chebyshev and Bielecki norms of the following problem:

$$
\begin{aligned}
& { }^{C} \mathcal{D}_{0^{+}}^{\kappa} \varsigma(\varkappa)=f(\varkappa, \varsigma(\varkappa), \varsigma(\mathfrak{h}(\varkappa)), \quad \varkappa \in[0, b], \kappa \in(0,1), \\
& \varsigma(0)=a_{0},
\end{aligned}
$$

where ${ }^{C} \mathcal{D}_{0^{+}}^{\kappa}$ is the Caputo FD of order $\kappa$.

Motivated by the preceding works, in the current paper, we investigate the existence of a unique solution and a UHML stability result for a tripled system of weighted Caputo fractional differential equations (TSWFDEs) with time delay

$$
\left\{\begin{array}{l}
{ }_{w}^{C} \mathcal{D}_{0^{+}}^{\kappa_{i}, \varphi} \varsigma_{i}(\varkappa) \\
\quad=f_{i}\left(\varkappa, \varsigma_{1}(\varkappa), \varsigma_{2}(\varkappa), \varsigma_{3}(\varkappa), \varsigma_{1}(\mathfrak{h}(\varkappa)), \varsigma_{2}\left(\mathfrak{h}(\varkappa), \varsigma_{3}(\mathfrak{h}(\varkappa)), \quad \varkappa \in J:=(0, b],\right.\right. \\
\varsigma_{i}(0)=a_{i} \in \mathbb{R}, \quad i=1,2,3, \\
\varsigma_{i}(\varkappa)=\vartheta_{i}(\varkappa), \quad \varkappa \in[-r, 0],
\end{array}\right.
$$

where ${ }_{w}^{C} \mathcal{D}_{0^{+}}^{\kappa_{i}, \varphi}$ is the weighted generalized Caputo fractional derivative (WCFD) of order $\kappa_{i} \in(0,1), w f_{i}:(0, b] \times \mathbb{R}^{3} \times \mathbb{R}^{3} \rightarrow \mathbb{R}$ is a given continuous function, $w(\varkappa) \neq 0$ is a weighted function with $w^{-1}(\varkappa)=\frac{1}{w(\varkappa)}, \varphi:(0, b] \rightarrow \mathbb{R}^{+}$is strictly increasing such that $\varphi \in C^{1}[0, b]$ with $\varphi^{\prime}(\varkappa) \neq 0$ for all $\varkappa \in(0, b], \vartheta_{i} \in C([-r, 0], \mathbb{R})$ and $\mathfrak{h} \in C([0, b],[-r, b])$ with $\mathfrak{h}(\varkappa) \leq \varkappa, r>0$. For simplicity, we denote the sequence of functions $\left(\varsigma_{1}(\varkappa), \varsigma_{2}(\varkappa), \varsigma_{3}(\varkappa)\right)$ by $\varsigma(\varkappa)$ and $\left(\varsigma_{1}(\mathfrak{h}(\varkappa)), \varsigma_{2}\left(\mathfrak{h}(\varkappa), \varsigma_{3}(\mathfrak{h}(\varkappa))\right.\right.$ by $\left.\varsigma^{(\mathfrak{h}}(\varkappa)\right)$. In the sequel, the functions such as $f_{i}\left(\varkappa, \varsigma_{1}(\varkappa), \varsigma_{2}(\varkappa), \varsigma_{3}(\varkappa), \varsigma_{1}(\mathfrak{h}(\varkappa)), \varsigma_{2}\left(\mathfrak{h}(\varkappa), \varsigma_{3}(\mathfrak{h}(\varkappa))\right.\right.$ will be written as $f_{i}(\varkappa, \varsigma(\varkappa), \varsigma(\mathfrak{h}(\varkappa)))$.

By a solution of system (1.1), it is meant that there is a sequence $\varsigma=\left(\varsigma_{1}, \varsigma_{2}, \varsigma_{3}\right)$ satisfying system $(1.1)$ on $(0, b]$.

The major contribution of this paper is to derive equivalent fractional integral equations to the (TSWFDEs) and to establish the existence of a unique solution and Ulam-HyersMittag-Leffler stability results for (TSWFDEs) with respect to Chebyshev and Bielecki 
norms with time delay. The Picard operator method and the Banach fixed point theorem are the important tools used to prove our main result. To the best of our observation, there is no analytical literature on studying the existence of tripled systems of fractional differential equations (TSWFDEs). This paper is the first work to study the existence of a unique solution and an Ulam-Hyers-Mittag-Leffler stability result for (TSWFDEs) with respect to Chebyshev and Bielecki norms with time delay.

This paper is systematized as follows: In Sect. 2, we render the rudimentary definitions and prove some lemmas that are applied throughout this paper, also we present the concepts of some fixed point theorems. In Sect. 3, we prove the existence of unique solutions and (UHML) stability results of system (1.1) under Chebyshev and Bielecki norms. In Sect. 4, we give a pertinent example to illustrate our results. Concluding remarks about our results are given in the last section.

\section{Preliminaries}

In this part, we give important definitions and auxiliary lemmas that are pertinent to our main results.

Let $E=C([-r, b], \mathbb{R})$ and $\Omega=C([0, b], \mathbb{R}),(b>0)$ be the Banach spaces of continuous function $u:[-r, b] \rightarrow \mathbb{R}$ and $v:[0, b] \rightarrow \mathbb{R}$ with the norms

$$
\|u\|_{E}=\max _{\varkappa \in[-r, b]}|u(\varkappa)|
$$

and

$$
\|v\|_{\Omega}=\max _{\varkappa \in[0, b]}|v(\varkappa)|
$$

respectively. Clearly, $E$ and $\Omega$ are the Banach spaces with the above norms. Let $E^{*}:=E \times$ $E \times E$ and $\Omega^{*}:=\Omega \times \Omega \times \Omega$ be the product spaces with the norms

$$
\left\|\left(u_{1}, u_{2}, u_{3}\right)\right\|_{E^{*}}=\sum_{i=1}^{3}\left\|u_{i}\right\|_{E}, \quad\left(u_{1}, u_{2}, u_{3}\right) \in E^{*}
$$

and

$$
\left\|\left(v_{1}, v_{2}, v_{3}\right)\right\|_{\Omega^{*}}=\sum_{i=1}^{3}\left\|v_{i}\right\|_{\Omega}, \quad\left(v_{1}, v_{2}, v_{3}\right) \in \Omega^{*},
$$

respectively.

Definition 2.1 ([1]) Let $\kappa>0$ and $\varsigma:[0, b] \rightarrow \mathbb{R}$ be an integrable function. Then (WCFI) and (WCFD) are given by

$$
{ }_{w} \mathcal{I}_{0^{+}}^{\kappa, \varphi} \varsigma(\varkappa)=\frac{w^{-1}(\varkappa)}{\Gamma(\kappa)} \int_{0}^{\varkappa} w(s) \mathcal{N}_{\varphi}^{\kappa-1}(\varkappa, s) \varsigma(s) d s
$$

and

$$
{ }_{w}^{C} \mathcal{D}_{0^{+}}^{\kappa, \varphi} \varsigma(\varkappa)=w^{-1}(\varkappa)\left(\frac{1}{\varphi^{\prime}(\varkappa)} \frac{d}{d \varkappa}\right)^{n}{ }_{w} \mathcal{I}_{0^{+}}^{n-\kappa, \varphi} \varsigma(\varkappa)
$$


respectively, where $\mathcal{N}_{\varphi}^{\kappa-1}(\varkappa, s)=\varphi^{\prime}(s)(\varphi(\varkappa)-\varphi(s))^{\kappa-1}, n=[\kappa]+1$, and $\varphi$ is a strictly increasing function on $[0, b]$.

Definition 2.2 ([1]) Let $\kappa \in(n-1, n), n \in \mathbb{N}$, and $\varsigma \in A_{w}^{n}[0, b]$. Then (WCFD) is given by

$$
{ }_{w}^{C} \mathcal{D}_{0^{+}}^{\kappa, \varphi} \varsigma(\varkappa)={ }_{w} \mathcal{D}_{0^{+}}^{\kappa, \varphi}\left[\varsigma(\varkappa)-w^{-1}(\varkappa) \sum_{i=1}^{n-1} \frac{\zeta_{\varphi, w}^{[i]}(0)}{i !}\left(\varphi(\varkappa)-\varphi(0)^{i}\right],\right.
$$

where $n=[\kappa]+1$ and $\varsigma_{\varphi, w}^{[i]}(\varkappa)=\left(\frac{1}{\varphi^{\prime}(\varkappa)} \frac{d}{d \varkappa}\right)^{i}(w(\varkappa) \varsigma(\varkappa))$. Moreover, ${ }^{C} \mathcal{D}_{0^{+}}^{\kappa, \varphi, w}$ can be written as

$$
\begin{aligned}
{ }_{w}^{C} \mathcal{D}_{0^{+}}^{\kappa, \varphi} \varsigma(\varkappa) & ={ }_{w} \mathcal{I}_{0^{+}}^{n-\kappa, \varphi} w^{-1}(\varkappa)\left(\frac{1}{\varphi^{\prime}(\varkappa)} \frac{d}{d \varkappa}\right)^{n} \varsigma(\varkappa) \\
& =\frac{w^{-1}(\varkappa)}{\Gamma(n-\kappa)} \int_{0}^{\varkappa} w(s) \mathcal{N}_{\varphi}^{\kappa-1}(\varkappa, s) \varsigma_{\varphi}^{[n]}(s) d s,
\end{aligned}
$$

where $n=[\kappa]+1$ and $\zeta_{\varphi}^{[n]}(\varkappa)=\left(\frac{1}{\varphi^{\prime}(\varkappa)} \frac{d}{d \varkappa}\right)^{n}(\varsigma(\varkappa))$. In particular, if $\kappa=n \in \mathbb{N}$, we have ${ }^{C} \mathcal{D}_{0^{+}}^{\kappa, \varphi, w} \varsigma(\varkappa)=\varsigma_{\varphi, w}^{[n]}(\varkappa)$.

Lemma 2.3 ([1]) Let $\kappa, \gamma>0$. Then

$$
{ }_{w} \mathcal{I}_{0^{+}}^{\kappa, \varphi}\left[w^{-1}(\varkappa)(\varphi(\varkappa)-\varphi(0))^{\gamma-1}\right]=\frac{\Gamma(\gamma)}{\Gamma(\kappa+\gamma)}(\varphi(\varkappa)-\varphi(0))^{\kappa+\gamma-1}
$$

and

$$
{ }_{w}^{C} \mathcal{D}_{0^{+}}^{\kappa, \varphi}\left[w^{-1}(\varkappa)(\varphi(\varkappa)-\varphi(0))^{\gamma-1}\right]=\frac{\Gamma(\gamma)}{\Gamma(\kappa-\gamma)}(\varphi(\varkappa)-\varphi(0))^{\kappa-\gamma-1}
$$

in case ${ }_{w}^{C} \mathcal{D}_{0^{+}}^{\kappa, \varphi}\left[w^{-1}(\varkappa)(\varphi(\varkappa)-\varphi(0))^{\gamma-1}\right]=0$ for all $\gamma-1 \in\{0,1, \ldots, n-1\}, n \in \mathbb{N}$.

Remark 2.4 ([1]) If $w(\varkappa)=1$, then equation (2.1) reduces to the relation obtained in Kilbas et al. [3].

Lemma 2.5 ([1]) For $\kappa \in(n-1, n), n \in \mathbb{N}$, we have

$$
{ }_{w}^{C} \mathcal{D}_{0^{+}}^{\kappa, \varphi} \mathcal{I}_{0^{+}}^{\kappa, \varphi} \varsigma(\varkappa)=\varsigma(\varkappa)
$$

and

$$
{ }_{w} \mathcal{I}_{0^{+}}^{\kappa, \varphi}{ }_{w} \mathcal{D}_{0^{+}}^{\kappa, \varphi} \varsigma(\varkappa)=\varsigma(\varkappa)-w^{-1}(\varkappa) \sum_{i=1}^{n-1} \frac{S_{\varphi, w}^{[i]}(0)}{i !}\left(\varphi(\varkappa)-\varphi(0)^{i}\right.
$$

In this paper, due to $\kappa \in(0,1)$, then

$$
{ }_{w} \mathcal{I}_{0^{+}}^{\kappa, \varphi}{ }_{w} \mathcal{D}_{0^{+}}^{\kappa, \varphi} \varsigma(\varkappa)=\varsigma(\varkappa)-\frac{1}{w(\varkappa)}(w(0) \varsigma(0)) .
$$

Lemma $2.6([1])$ Let $\kappa>0$. Then ${ }_{w} \mathcal{I}_{0^{+}}^{\kappa, \varphi}:(C[0, b], \mathbb{R}) \rightarrow(C[0, b], \mathbb{R})$ is bounded. Moreover,

$$
{ }_{w} \mathcal{I}_{0^{+}}^{\kappa, \varphi} \varsigma(\varkappa)=\lim _{\varkappa \rightarrow 0^{+}} \mathcal{I}_{0^{+}}^{\kappa, \varphi, w} \varsigma(\varkappa)=0 .
$$


Definition 2.7 ([35]) Let $(X, d)$ be a metric space. Now $T: X \rightarrow X$ is a Picard operator if there exists $\varsigma^{*} \in X$ such that $F_{T}=\varsigma^{*}$, where $F_{T}=\{\varsigma \in X: T(\varsigma)=\varsigma\}$ is the fixed point set of $T$, and the sequence $\left(T^{n}\left(\varsigma_{0}\right)\right)_{n \in \mathbb{N}}$ converges to $\varsigma^{*}$ for all $\varsigma_{0} \in X$.

Lemma 2.8 ([35]) Let $(X, d, \leq)$ be an ordered metric space, and let $T: X \rightarrow X$ be an increasing Picard operator with $F_{T}=\left\{\varsigma_{T}^{*}\right\}$. Then, for $\varsigma \in X, \varsigma \leq T(\varsigma)$ implies $\varsigma \leq \varsigma_{T}^{*}$.

Remark 2.9 A sequence $\left(\widehat{\zeta}_{1}, \widehat{\zeta}_{2}, \widehat{\zeta}_{3}\right) \in E^{*}$ satisfies the inequality

$$
\begin{aligned}
& \mid{ }_{w}^{C} \mathcal{D}_{0^{+}}^{\kappa_{i}, \varphi} \widehat{\zeta}_{i}(\varkappa)-f_{i}(\varkappa, \widehat{\zeta}, \widehat{\zeta}(\mathfrak{h}(\varkappa)) \mid \\
& \quad \leq \varepsilon_{i} E_{\kappa_{i}}(\varphi(\varkappa)-\varphi(0))^{\kappa_{i}}, \quad i=1,2, \ldots, n, \varkappa \in(0, b],
\end{aligned}
$$

if and only if there exists a function $w \eta_{i} \in \Omega$ such that

(1) $\left|w(\varkappa) \eta_{i}(\varkappa)\right| \leq \varepsilon_{i} E_{\kappa_{i}}(\varphi(\varkappa)-\varphi(0))^{\kappa_{i}}, \varkappa \in(0, b]$;

(2) ${ }_{w}^{C} \mathcal{D}_{0^{+}}^{\kappa, \varphi} \widehat{\zeta}_{i}(\varkappa)=f_{i}(\varkappa, \widehat{\zeta}, \widehat{\zeta}(\mathfrak{h}(\varkappa)))+\eta_{i}(\varkappa), \varkappa \in(0, b]$.

Definition 2.10 System (1.1) is (UHML) stable with respect to $E_{\kappa}\left((\varphi(\varkappa)-\varphi(0))^{\kappa}\right)=$ $\max _{\varkappa \in(0, b]}\left\{E_{\kappa_{i}}(\varphi(\varkappa)-\varphi(0))^{\kappa_{i}}\right\}, i=1,2,3$, if there exists $\mathcal{M}>0$ such that, for each $\varepsilon=$ $\max \left\{\varepsilon_{1}, \varepsilon_{2}, \varepsilon_{3}\right\}>0$ and each sequence $\left(\widehat{\zeta}_{1}, \widehat{\zeta}_{2}, \widehat{\zeta}_{3}\right) \in E^{*}$ satisfies inequality (2.3), there exists a solution $\left(\varsigma_{1}, \varsigma_{2}, \varsigma_{3}\right) \in E^{*}$ of system (1.1) with

$$
\left\|\left(\widehat{\varsigma_{1}}, \widehat{\varsigma}_{2}, \widehat{\varsigma}_{3}\right)-\left(\varsigma_{1}, \varsigma_{2}, \varsigma_{3}\right)\right\|_{E^{*}} \leq \mathcal{M} \varepsilon E_{\kappa}(\varphi(\varkappa)-\varphi(0))^{\kappa}, \quad \varkappa \in(0, b] .
$$

Lemma 2.11 Let $i=1,2,3$ and $w f_{i}:(0, b] \times \mathbb{R}^{3} \times \mathbb{R}^{3} \rightarrow \mathbb{R}$ be continuous functions. If the sequence $\left(\varsigma_{1}, \varsigma_{2}, \varsigma_{3}\right) \in E^{*}$ satisfies system (1.1), then, in view of Lemma 2.5 , we can easily prove that $\left(\varsigma_{1}, \varsigma_{2}, \varsigma_{3}\right)$ satisfies the following integral equations:

$$
\varsigma_{i}(\varkappa)=\left\{\begin{array}{l}
\frac{w(0)}{w(\varkappa)} a_{i}+{ }_{w} \mathcal{I}^{\kappa_{i}, \varphi} f_{i}(s, \varsigma(s), \varsigma(\mathfrak{h}(s)))(\varkappa), \quad \varkappa \in(0, b], \\
\vartheta_{i}(\varkappa), \quad \varkappa \in[-r, 0],
\end{array}\right.
$$

for all $i=1,2,3$.

Lemma 2.12 Let $\kappa_{i} \in(0,1), i=1,2,3$, and $\mu=\max _{\varkappa \in(0, b]}|w(\varkappa)|$. If a sequence $\left(\widehat{\zeta}_{1}, \widehat{\zeta}_{2}, \widehat{\zeta}_{3}\right) \in$ $E^{*}$ satisfies inequality (2.3), then $\left(\widehat{\zeta}_{1}, \widehat{\zeta}_{2}, \widehat{\zeta}_{3}\right)$ satisfies the following integral inequality:

$$
\begin{aligned}
& \left|\widehat{\zeta}_{i}(\varkappa)-\frac{w(0)}{w(\varkappa)} a_{i}-\frac{w^{-1}(\varkappa)}{\Gamma\left(\kappa_{i}\right)} \int_{0}^{\varkappa} w(s) \mathcal{N}_{\varphi}^{\kappa_{i}-1}(\varkappa, s) f_{i}(s, \widehat{\zeta}(s), \widehat{\zeta}(\mathfrak{h}(s))) d s\right| \\
& \quad \leq \varepsilon_{i} E_{\kappa_{i}}(\varphi(\varkappa)-\varphi(0))^{\kappa_{i}} .
\end{aligned}
$$

Proof Let $\left(\widehat{\varsigma}_{1}, \widehat{\varsigma}_{2}, \widehat{\zeta}_{3}\right) \in E^{*}$ satisfy inequality (2.3). Then, in the light of Remark 2.9 and Lemma 2.11, we have

$$
\begin{aligned}
\widehat{\zeta}_{i}(\varkappa) & =\frac{w(0)}{w(\varkappa)} a_{i}+{ }_{w} \mathcal{I}^{\kappa_{i}, \varphi}\left[f_{i}(s, \varsigma(s), \varsigma(\mathfrak{h}(s)))+\eta_{i}(s)\right](\varkappa) \\
& =\frac{w(0)}{w(\varkappa)} a_{i}+\frac{w^{-1}(\varkappa)}{\Gamma\left(\kappa_{i}\right)} \int_{0}^{\varkappa} w(s) \mathcal{N}_{\varphi}^{\kappa_{i}-1}(\varkappa, s) f_{i}(s, \widehat{\zeta}(s), \widehat{\zeta}(\mathfrak{h}(s))) d s
\end{aligned}
$$




$$
+\frac{w^{-1}(\varkappa)}{\Gamma\left(\kappa_{i}\right)} \int_{0}^{\varkappa} w(s) \mathcal{N}_{\varphi}^{\kappa_{i}-1}(\varkappa, s)\left|\left(\eta_{i}(s)\right)\right| d s
$$

Thus, we have

$$
\begin{aligned}
\mid \widehat{\zeta}_{i}(\varkappa) & -\frac{w(0)}{w(\varkappa)} a_{i}-\frac{w^{-1}(\varkappa)}{\Gamma\left(\kappa_{i}\right)} \int_{0}^{\varkappa} w(s) \mathcal{N}_{\varphi}^{\kappa_{i}-1}(\varkappa, s) f_{i}(s, \widehat{\zeta}(s), \widehat{\zeta}(\mathfrak{h}(s))) d s \mid \\
& \leq \frac{w^{-1}(\varkappa)}{\Gamma\left(\kappa_{i}\right)} \int_{0}^{\varkappa} \mathcal{N}_{\varphi}^{\kappa_{i}-1}(\varkappa, s)\left|w(s)\left(\eta_{i}(s)\right)\right| d s \\
& \leq \varepsilon_{i} \frac{w^{-1}(\varkappa)}{\Gamma\left(\kappa_{i}\right)} \int_{0}^{\varkappa} \mathcal{N}_{\varphi}^{\kappa_{i}-1}(\varkappa, s) E_{\kappa_{i}}(\varphi(s)-\varphi(0))^{\kappa_{i}} d s \\
& \leq \frac{\varepsilon_{i}}{\mu} \sum_{n=1}^{\infty} \frac{1}{\Gamma\left(n \kappa_{i}+1\right)} \frac{1}{\Gamma\left(\kappa_{i}\right)} \int_{0}^{\varkappa} \mathcal{N}_{\varphi}^{\kappa_{i}-1}(\varkappa, s)(\varphi(s)-\varphi(0))^{n \kappa_{i}} d s \\
& \leq \frac{\varepsilon_{i}}{\mu} \sum_{n=1}^{\infty} \frac{(\varphi(\varkappa)-\varphi(0))^{(n+1) \kappa_{i}}}{\Gamma\left((n+1) \kappa_{i}+1\right)} \\
& <\frac{\varepsilon_{i}}{\mu} \sum_{n=1}^{\infty} \frac{(\varphi(\varkappa)-\varphi(0))^{n \kappa_{i}}}{\Gamma\left(n \kappa_{i}+1\right)}<\frac{\varepsilon_{i}}{\mu} E_{\kappa_{i}}(\varphi(\varkappa)-\varphi(0))^{\kappa_{i}} .
\end{aligned}
$$

Let us consider the continuous operator $\mathcal{G}: E^{*} \rightarrow E^{*}$ defined by

$$
\mathcal{G}(\varsigma)(\varkappa)=\left(\mathcal{G}_{1}(\varsigma)(\varkappa), \mathcal{G}_{2}(\varsigma)(\varkappa), \mathcal{G}_{3}(\varsigma)(\varkappa)\right)
$$

where

$$
\begin{aligned}
& \mathcal{G}_{1}(\varsigma)(\varkappa)= \begin{cases}\frac{w(0)}{w(\varkappa)} a_{1}+{ }_{w} \mathcal{I}^{\kappa_{1}, \varphi} f_{1}(s, \varsigma(s), \varsigma(\mathfrak{h}(s)))(\varkappa), & \varkappa \in(0, b], \\
\vartheta_{1}(\varkappa), \quad \varkappa \in[-r, 0],\end{cases} \\
& \mathcal{G}_{2}(\varsigma)(\varkappa)= \begin{cases}\frac{w(0)}{w(\varkappa)} a_{2}+{ }_{w} \mathcal{I}^{\kappa_{2}, \varphi} f_{2}(s, \varsigma(s), \varsigma(\mathfrak{h}(s)))(\varkappa), & \varkappa \in(0, b], \\
\vartheta_{2}(\varkappa), & \varkappa \in[-r, 0],\end{cases}
\end{aligned}
$$

and

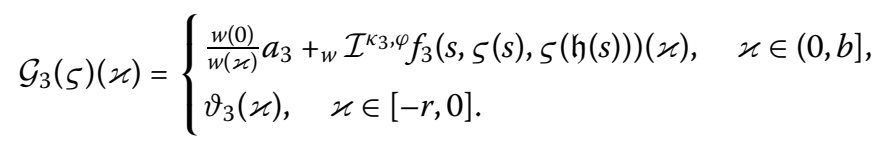

\section{Main results}

In this section, we prove the existence of a unique solution and a (UHML) stability result for system (1.1) with respect to Chebyshev and Bielecki norms with time delay. For our analysis, the following hypotheses should be satisfied.

(H1) $w f_{i} \in C\left([0, b] \times \mathbb{R}^{3} \times \mathbb{R}^{3}, \mathbb{R}\right), \mathfrak{h} \in C([0, b],[-r, b]), \mathfrak{h}(\varkappa) \leq \varkappa, r>0, i=1,2,3$.

(H1) $\left(w f_{i}\right):[0, b] \times \mathbb{R}^{3} \times \mathbb{R}^{3} \rightarrow \mathbb{R}$ are continuous functions and there exists $\mathcal{L}_{i}>0$ such that

$$
\left|w(\varkappa) f_{i}(\varkappa, \varsigma, z)-w(\varkappa) f_{i}(\varkappa, v, y)\right| \leq \mathcal{L}_{i} \sum_{k=1}^{3}\left[\left|\varsigma_{k}-v_{k}\right|+\left|z_{k}-y_{k}\right|\right]
$$

for all $\varkappa \in(0, b], \varsigma, v, z, y \in \mathbb{R}^{3}$. 
Theorem 3.1 Assume that (H1) and (H2) are satisfied. If

$$
\Upsilon=\sum_{i=1}^{3} \frac{2 \mathcal{L}_{i}}{\mu} \frac{(\varphi(b)-\varphi(0))^{\kappa_{i}}}{\Gamma\left(\kappa_{i}+1\right)}<1,
$$

then system (1.1) has a unique solution $\varsigma=\left(\varsigma_{1}, \varsigma_{2}, \varsigma_{3}\right) \in \Xi:=E \cap \Omega \times E \cap \Omega \times E \cap \Omega$.

Proof Define a closed ball set as $\mathbb{P}_{\zeta}=\left\{\left(\varsigma_{1}, \varsigma_{2}, \varsigma_{3}\right) \in E^{*}:\left\|\left(\varsigma_{1}, \varsigma_{2}, \varsigma_{3}\right)\right\|_{E^{*}} \leq \zeta\right\}$ with $\zeta \geq \frac{\Lambda}{1-\Upsilon}$, where

$$
\Lambda:=\sum_{i=1}^{3}\left[\frac{|w(0)|}{\mu} a_{i}+\frac{2 \mathcal{L}_{i}}{\mu} \widehat{f}_{i} \frac{(\varphi(b)-\varphi(0))^{\kappa_{i}}}{\Gamma\left(\kappa_{i}+1\right)}\right],
$$

and $\widehat{f_{i}}=\sup _{s \in[0, b]}\left|w f_{i}(s, 0,0)\right|, \mu=\max _{\varkappa \in[0, b]}|w(\varkappa)|$. In order to examine the existence of a unique solution by means of the Banach fixed point theorem, we only prove that the operator $\mathcal{G}(\varsigma)$ defined by $(2.5)$ has a fixed point in $\Xi$. For this purpose, we split the proof into the following steps.

Step (1): $\mathcal{G}(\varsigma)$ is continuous.

From the continuity of functions $w f_{i}(\varkappa, \varsigma(\varkappa), \varsigma(\mathfrak{h}(\varkappa)))$, we deduce that the operator $\mathcal{G}\left(\varsigma_{1}, \varsigma_{2}, \varsigma_{3}\right)$ is continuous too.

Step (2): $\mathcal{G}(\varsigma) \in \mathbb{P}_{\zeta}$

Case (1). For each $\varsigma=\left(\varsigma_{1}, \varsigma_{2}, \varsigma_{3}\right) \in \mathbb{P}_{\zeta}$ and for $\varkappa \in[-r, 0]$, we have

$$
\left\|\mathcal{G}_{i}\left(\varsigma_{1}, \varsigma_{2}, \varsigma_{3}\right)\right\|_{E}=\left\|\vartheta_{i}\right\|_{E}, \quad i=1,2,3
$$

Hence

$$
\begin{aligned}
\left\|\mathcal{G}\left(\varsigma_{1}, \varsigma_{2}, \varsigma_{3}\right)\right\|_{E^{*}} & \leq\left\|\vartheta_{1}\right\|_{E}+\left\|\vartheta_{2}\right\|_{E}+\left\|\vartheta_{3}\right\|_{E} \\
& \leq\left\|\left(\vartheta_{1}, \vartheta_{2}, \vartheta_{3}\right)\right\|_{E^{*}} \leq \zeta .
\end{aligned}
$$

Case (2). For each $\varsigma=\left(\varsigma_{1}, \varsigma_{2}, \varsigma_{3}\right) \in \mathbb{P}_{\zeta}$ and for $\varkappa \in[0, b]$, we have

$$
\begin{aligned}
\left|\mathcal{G}_{i}\left(\varsigma_{1}, \varsigma_{2}, \varsigma_{3}\right)\right| & \\
\leq & \frac{|w(0)|}{|w(\varkappa)|} a_{i}+\frac{w^{-1}(\varkappa)}{\Gamma(\kappa)} \int_{0}^{\varkappa} \mathcal{N}_{\varphi}^{\kappa-1}(\varkappa, s) \\
& \times\left\{\left|w(s)\left(f_{i}(s, \varsigma(s), \varsigma(\mathfrak{h}(s)))-f_{i}(s, 0,0)\right)\right|+\left|w(s) f_{i}(s, 0,0)\right|\right\} d s \\
\leq & \frac{|w(0)|}{\mu} a_{i}+\frac{\mathcal{L}_{i}}{\mu \Gamma(\kappa)} \int_{0}^{\varkappa} \mathcal{N}_{\varphi}^{\kappa-1}(\varkappa, s)\left\{\left|\varsigma_{1}(s)\right|+\left|\varsigma_{2}(s)\right|+\left|\varsigma_{3}(s)\right|\right. \\
& +\mid \varsigma_{1}\left(\mathfrak { h } ( s ) | + | \varsigma _ { 2 } \left(\mathfrak{h}(s)|+| \varsigma_{3}\left(\mathfrak{h}(s)|+| w(s) f_{i}(s, 0,0) \mid\right\} d s\right.\right. \\
\leq & \frac{|w(0)|}{\mu} a_{i}+\frac{2 \mathcal{L}_{i}}{\mu \Gamma\left(\kappa_{i}\right)}\left\{\left(\left\|\varsigma_{1}\right\|_{E}+\left\|\varsigma_{2}\right\|_{E}+\left\|\varsigma_{3}\right\|_{E}\right)+\widehat{f}_{i}\right\} \int_{0}^{\varkappa} \mathcal{N}_{\varphi}^{\kappa-1}(\varkappa, s) d s \\
\leq & \frac{|w(0)|}{\mu} a_{i}+\frac{2 \mathcal{L}_{i}}{\mu}\left\{\left\|\left(\varsigma_{1}, \varsigma_{2}, \varsigma_{3}\right)\right\|_{E^{*}}+\widehat{f}_{i}\right\} \frac{(\varphi(\varkappa)-\varphi(0))^{\kappa_{i}}}{\Gamma\left(\kappa_{i}+1\right)} \\
\leq & \frac{|w(0)|}{\mu} a_{i}+\frac{2 \mathcal{L}_{i}}{\mu}\left\{\zeta+\widehat{f}_{i}\right\} \frac{(\varphi(\varkappa)-\varphi(0))^{\kappa_{i}}}{\Gamma\left(\kappa_{i}+1\right)}
\end{aligned}
$$




$$
\leq \frac{|w(0)|}{\mu} a_{i}+\frac{2 \mathcal{L}_{i}}{\mu} \widehat{f}_{i} \frac{(\varphi(b)-\varphi(0))^{\kappa_{i}}}{\Gamma\left(\kappa_{i}+1\right)}+\frac{2 \mathcal{L}_{i}}{\mu} \frac{(\varphi(b)-\varphi(0))^{\kappa_{i}}}{\Gamma\left(\kappa_{i}+1\right)} \zeta
$$

which implies

$$
\begin{aligned}
\left\|\mathcal{G}\left(\varsigma_{1}, \varsigma_{2}, \varsigma_{3}\right)\right\|_{E^{*}} \\
\quad=\left\|\mathcal{G}_{1}\left(\varsigma_{1}, \varsigma_{2}, \varsigma_{3}\right)\right\|_{E}+\left\|\mathcal{G}_{2}\left(\varsigma_{1}, \varsigma_{2}, \varsigma_{3}\right)\right\|_{E}+\left\|\mathcal{G}_{3}\left(\varsigma_{1}, \varsigma_{2}, \varsigma_{3}\right)\right\|_{E} \\
\quad \leq \sum_{i=1}^{3}\left[\frac{|w(0)|}{\mu} a_{i}+\frac{2 \mathcal{L}_{i} \widehat{f}_{i}(\varphi(b)-\varphi(0))^{\kappa_{i}}}{\Gamma\left(\kappa_{i}+1\right)}\right]+\sum_{i=1}^{3} \frac{2 \mathcal{L}_{i}}{\mu} \frac{(\varphi(b)-\varphi(0))^{\kappa_{i}}}{\Gamma\left(\kappa_{i}+1\right)} \zeta \\
\quad<\Lambda+\zeta \Upsilon \leq \zeta .
\end{aligned}
$$

This proves that $\mathcal{G}(\varsigma) \in \mathbb{P}_{\zeta}$.

Step (3): $\mathcal{G}(\varsigma)$ is a contraction in $\mathbb{P}_{\zeta}$.

In this step, we will show that the operator $\mathcal{G}(\varsigma)$ is a contraction mapping on $\mathbb{P}_{\zeta}$ with respect to the norm $\left\|\left(\varsigma_{1}, \varsigma_{2}, \varsigma_{3}\right)\right\|_{E^{*}}=\left\|\varsigma_{1}\right\|_{E}+\left\|\varsigma_{2}\right\|_{E}+\left\|\varsigma_{3}\right\|_{E}$.

Case (1): For $\left(\varsigma_{1}, \varsigma_{2}, \varsigma_{3}\right),\left(v_{1}, v_{2}, v_{3}\right) \in \mathbb{P}_{\zeta}$ and $\varkappa \in[-r, 0]$, we have

$$
\left\|\mathcal{G}_{i}\left(\varsigma_{1}, \varsigma_{2}, \varsigma_{3}\right)-\mathcal{G}_{i}\left(v_{1}, v_{2}, v_{3}\right)\right\|_{E}=0, \quad i=1,2,3
$$

It follows that

$$
\begin{aligned}
\left\|\mathcal{G}\left(\varsigma_{1}, \varsigma_{2}, \varsigma_{3}\right)-\mathcal{G}\left(v_{1}, v_{2}, v_{3}\right)\right\|_{E^{*}} & =\sum_{i=1}^{3}\left\|\mathcal{G}_{i}\left(\varsigma_{1}, \varsigma_{2}, \varsigma_{3}\right)-\mathcal{G}_{i}\left(v_{1}, v_{2}, v_{3}\right)\right\|_{E} \\
& =0 \leq \Upsilon\left\|\left(\varsigma_{1}, \varsigma_{2}, \varsigma_{3}\right)-\left(v_{1}, \nu_{2}, v_{3}\right)\right\|_{E^{*}} .
\end{aligned}
$$

Case (2): For $\left(\varsigma_{1}, \varsigma_{2}, \varsigma_{3}\right),\left(v_{1}, v_{2}, v_{3}\right) \in \mathbb{P}_{\zeta}, \varkappa \in(0, b]$ and by $\left(\mathrm{H}_{2}\right)$, we obtain

$$
\begin{aligned}
\left\|\mathcal{G}_{i}\left(\varsigma_{1}, \varsigma_{2}, \varsigma_{3}\right)-\mathcal{G}_{i}\left(v_{1}, v_{2}, v_{3}\right)\right\|_{E} \\
\leq \frac{w^{-1}(\varkappa)}{\Gamma\left(\kappa_{i}\right)} \int_{0}^{\varkappa} w(s) \mathcal{N}_{\varphi}^{\kappa_{i}-1}(\varkappa, s)\left|\left[f_{i}(s, \varsigma(s), \varsigma(\mathfrak{h}(s)))-f_{i}(s, v(s), v(\mathfrak{h}(s)))\right]\right| d s \\
\leq \frac{w^{-1}(\varkappa)}{\Gamma\left(\kappa_{i}\right)} \int_{0}^{\varkappa} \mathcal{N}_{\varphi}^{\kappa_{i}-1}(\varkappa, s)\left|w(s)\left[f_{i}(s, \varsigma(s), \varsigma(\mathfrak{h}(s)))-f_{i}(s, v(s), v(\mathfrak{h}(s)))\right]\right| d s \\
\leq \frac{\mathcal{L}_{i}}{\mu \Gamma\left(\kappa_{i}\right)} \int_{0}^{\varkappa} \mathcal{N}_{\varphi}^{\kappa_{i}-1}(\varkappa, s)\left\{\left|\varsigma_{1}(s)-v_{1}(s)\right|+\left|\varsigma_{2}(s)-v_{2}(s)\right|+\left|\varsigma_{3}(s)-v_{3}(s)\right|\right. \\
\left.\quad+\left|\varsigma_{1}(\mathfrak{h}(s))-v_{1}(\mathfrak{h}(s))\right|+\left|\varsigma_{2}(\mathfrak{h}(s))-v_{2}(\mathfrak{h}(s))\right|+\left|\varsigma_{3}(\mathfrak{h}(s))-v_{3}(\mathfrak{h}(s))\right|\right\} d s \\
\leq \frac{2 \mathcal{L}_{i}}{\mu} \frac{(\varphi(b)-\varphi(0))^{\kappa_{i}}}{\Gamma\left(\kappa_{i}+1\right)}\left[\left\|\varsigma_{1}-v_{1}\right\|_{E}+\left\|\varsigma_{2}-v_{2}\right\|_{E}+\left\|\varsigma_{3}-v_{3}\right\|_{E}\right] \\
=\frac{2 \mathcal{L}_{i}}{\mu} \frac{(\varphi(b)-\varphi(0))^{\kappa_{i}}}{\Gamma\left(\kappa_{i}+1\right)}\left\|\left(\varsigma_{1}, \varsigma_{2}, \varsigma_{3}\right)-\left(v_{1}, v_{2}, v_{3}\right)\right\|_{E^{*}} .
\end{aligned}
$$

It follows that

$$
\begin{aligned}
& \left\|\mathcal{G}\left(\varsigma_{1}, \varsigma_{2}, \varsigma_{3}\right)-\mathcal{G}\left(v_{1}, v_{2}, v_{3}\right)\right\|_{E^{*}} \\
& \quad=\sum_{i=1}^{3}\left\|\mathcal{G}_{i}\left(\varsigma_{1}, \varsigma_{2}, \varsigma_{3}\right)-\mathcal{G}_{i}\left(v_{1}, v_{2}, v_{3}\right)\right\|_{E}
\end{aligned}
$$




$$
\begin{aligned}
& \leq \sum_{i=1}^{3} \frac{2 \mathcal{L}_{i}}{\mu} \frac{(\varphi(b)-\varphi(0))^{\kappa_{i}}}{\Gamma\left(\kappa_{i}+1\right)}\left\|\left(\varsigma_{1}, \varsigma_{2}, \varsigma_{3}\right)-\left(v_{1}, v_{2}, v_{3}\right)\right\|_{E^{*}} \\
& \leq \Upsilon\left\|\left(\varsigma_{1}, \varsigma_{2}, \varsigma_{3}\right)-\left(v_{1}, \nu_{2}, \nu_{3}\right)\right\|_{E^{*}} .
\end{aligned}
$$

Thus, in light of the above cases, for all $\varkappa \in[-r, b]$, we get

$$
\left\|\mathcal{G}\left(\varsigma_{1}, \varsigma_{2}, \ldots, \varsigma_{n}\right)-\mathcal{G}\left(v_{1}, v_{2}, \ldots, v_{n}\right)\right\|_{E^{*}} \leq \Upsilon\left\|\left(\varsigma_{1}, \varsigma_{2}, \varsigma_{3}\right)-\left(v_{1}, v_{2}, v_{3}\right)\right\|_{E^{*}}
$$

Thus, the operator $\mathcal{G}\left(\varsigma_{1}, \varsigma_{2}, \varsigma_{3}\right)$ is a contraction mapping on $\Xi$ with respect to the norm

$$
\left\|\left(\varsigma_{1}, \varsigma_{2}, \varsigma_{3}\right)\right\|_{E^{*}}=\left\|\varsigma_{1}\right\|_{E}+\left\|\varsigma_{2}\right\|_{E}+\left\|\varsigma_{3}\right\|_{E} .
$$

So, by the above steps and the Banach fixed point theorem, we deduce that system (1.1) has a unique solution in $\Xi$.

Theorem 3.2 Assume that $(\mathrm{H} 1)$ and $(\mathrm{H} 2)$ are satisfied. If

$$
\Upsilon=\sum_{i=1}^{3} \frac{2 \mathcal{L}_{i}}{\mu} \frac{(\varphi(b)-\varphi(0))^{\kappa_{i}}}{\Gamma\left(\kappa_{i}+1\right)}<1
$$

and $\mathcal{M}=\frac{3}{\mu \lambda}>0$, where $\lambda=1-\sum_{i=1}^{3} \frac{2 \mathcal{L}_{i}(\varphi(b)-\varphi(0))^{\kappa_{i}}}{\mu \Gamma\left(\kappa_{i}+1\right)} \neq 0$, then

$$
{ }_{w}^{C} \mathcal{D}_{0^{+}}^{\kappa_{i}, \varphi} S_{i}(\varkappa)=f_{i}(\varkappa, \varsigma(\varkappa), \varsigma(\mathfrak{h}(\varkappa))), \quad \varkappa \in(0, b], i=1,2,3
$$

is UHML stable.

Proof Let $\widehat{\varsigma}=\left(\widehat{\varsigma}_{1}, \widehat{\varsigma}_{2}, \widehat{\zeta}_{3}\right) \in \Xi$ be a sequence satisfying inequality $(2.3)$ and $\varsigma=\left(\varsigma_{1}, \varsigma_{2}, \varsigma_{3}\right) \in$ $\Xi$ be a unique solution to the following (TSWFDEs)

$$
\begin{aligned}
& { }_{w}^{C} \mathcal{D}_{0^{+}}^{\kappa_{i}, \varphi} S_{i}(\varkappa)=f_{i}(\varkappa, \zeta(\varkappa), \varsigma(\mathfrak{h}(\varkappa))), \quad \varkappa \in(0, b], \\
& \varsigma_{i}(\varkappa)=\widehat{\zeta}_{i}(\varkappa), \quad \varkappa \in[-r, 0] .
\end{aligned}
$$

Now, by Lemma 2.11, for $i=1,2,3$, we have

$$
S_{i}(\varkappa)=\left\{\begin{array}{l}
\frac{w(0)}{w(\varkappa)} a_{i}+\frac{w^{-1}(\varkappa)}{\Gamma\left(\kappa_{i}\right)} \int_{0}^{\varkappa} w(s) \mathcal{N}_{\varphi}^{\kappa_{i}-1}(\varkappa, s) f_{i}(s, \varsigma(s), \varsigma(\mathfrak{h}(s))) d s, \quad \varkappa \in(0, b], \\
\widehat{\zeta}_{i}(\varkappa), \quad \varkappa \in[-r, 0] .
\end{array}\right.
$$

First, for all $\varkappa \in[-r, 0]$, we have $\left|\widehat{\varsigma}_{i}(\varkappa)-\varsigma_{i}(\varkappa)\right|=0$. Next, from $\left(\mathrm{H}_{2}\right)$ and Lemma 2.12, for each $\varkappa \in(0, b]$, we have

$$
\begin{aligned}
\left|\widehat{\zeta}_{i}(\varkappa)-\varsigma_{i}(\varkappa)\right| & \\
\leq & \left|\widehat{\zeta}_{i}(\varkappa)-\frac{w(0)}{w(\varkappa)} a_{i}-\frac{w^{-1}(\varkappa)}{\Gamma\left(\kappa_{i}\right)} \int_{0}^{\varkappa} w(s) \mathcal{N}_{\varphi}^{\kappa_{i}-1}(\varkappa, s) f_{i}(s, \widehat{\zeta}(s), \widehat{\zeta}(\mathfrak{h}(s))) d s\right| \\
& +\mid \frac{w^{-1}(\varkappa)}{\Gamma\left(\kappa_{i}\right)} \int_{0}^{\varkappa} w(s) \mathcal{N}_{\varphi}^{\kappa_{i}-1}(\varkappa, s) f_{i}(s, \widehat{\zeta}(s), \widehat{\zeta}(\mathfrak{h}(s))) d s
\end{aligned}
$$




$$
\begin{aligned}
& -\frac{w^{-1}(\varkappa)}{\Gamma\left(\kappa_{i}\right)} \int_{0}^{\varkappa} w(s) \mathcal{N}_{\varphi}^{\kappa_{i}-1}(\varkappa, s) f_{i}(s, \varsigma(s), \varsigma(\mathfrak{h}(s))) d s \mid \\
\leq & \frac{\varepsilon_{i}}{\mu} E_{\kappa_{i}}(\varphi(\varkappa)-\varphi(0))^{\kappa_{i}} \\
& +\frac{w^{-1}(\varkappa)}{\Gamma\left(\kappa_{i}\right)} \int_{0}^{\varkappa} \mathcal{N}_{\varphi}^{\kappa_{i}-1}(\varkappa, s)\left|w(s)\left[f_{i}(s, \widehat{\zeta}(s), \widehat{\zeta}(\mathfrak{h}(s)))-f_{i}(s, \varsigma(s), \varsigma(\mathfrak{h}(s)))\right]\right| d s \\
\leq & \frac{\varepsilon_{i}}{\mu} E_{\kappa_{i}}(\varphi(\varkappa)-\varphi(0))^{\kappa_{i}} \\
& +\frac{\mathcal{L}_{i} w^{-1}(\varkappa)}{\Gamma\left(\kappa_{i}\right)} \int_{0}^{\varkappa} \mathcal{N}_{\varphi}^{\kappa_{i}-1}(\varkappa, s)\left[\left|\widehat{\zeta}_{1}(s)-\varsigma_{1}(s)\right|+\left|\widehat{\zeta}_{2}(s)-\varsigma_{2}(s)\right|+\left|\widehat{\zeta}_{3}(s)-\varsigma_{3}(s)\right|\right. \\
& \left.\times\left|\widehat{\zeta}_{1}(\mathfrak{h}(s))-\varsigma_{1}(\mathfrak{h}(s))\right|+\left|\widehat{\zeta}_{2}(\mathfrak{h}(s))-\varsigma_{2}(\mathfrak{h}(s))\right|+\left|\widehat{\zeta}_{3}(\mathfrak{h}(s))-\varsigma_{3}(\mathfrak{h}(s))\right|\right] d s .
\end{aligned}
$$

Set $\Omega_{+}:=C\left([-r, b], \mathbb{R}^{+}\right)$and $\Omega_{+}^{*}=\Omega_{+} \times \Omega_{+} \times \Omega_{+}$. Now, for each sequence $y=\left(y_{1}, y_{2}, y_{3}\right) \in$ $\Omega_{+}^{*}$, we consider the operator $\mathcal{U}: \Omega_{+}^{*} \rightarrow \Omega_{+}$defined by

$$
\mathcal{U}\left(y_{1}, y_{2}, y_{3}\right)=\left(\mathcal{U}_{1}\left(y_{1}, y_{2}, y_{3}\right)(\varkappa), \mathcal{U}_{2}\left(y_{1}, y_{2}, y_{3}\right)(\varkappa), \mathcal{U}_{3}\left(y_{1}, y_{2}, \ldots, y_{n}\right)(\varkappa)\right)
$$

where $\mathcal{U}_{i}\left(y_{1}, y_{2}, y_{3}\right)(\varkappa), i=1,2,3$, given as

$$
\begin{aligned}
& \mathcal{U}_{i}\left(y_{1}, y_{2}, y_{3}\right)(\varkappa) \\
& =\left\{\begin{array}{c}
\frac{\varepsilon_{i}}{\mu} E_{\kappa_{i}}(\varphi(\varkappa)-\varphi(0))^{\kappa_{i}}+\frac{\mathcal{L}_{i} w^{-1}(\varkappa)}{\Gamma\left(\kappa_{i}\right)} \int_{0}^{\varkappa} \mathcal{N}_{\varphi}^{\kappa_{i}-1}(\varkappa, s) y_{1}(s) d s \\
\quad+\frac{\mathcal{L}_{i} w^{-1}(\varkappa)}{\Gamma\left(\kappa^{\prime}\right)} \int_{0}^{\varkappa} \mathcal{N}_{\varphi}^{\kappa_{i}-1}(\varkappa, s) y_{2}(s) d s+\frac{\mathcal{L}_{i} w^{-1}(\varkappa)}{\Gamma\left(\kappa_{i}\right)} \int_{0}^{\varkappa} \mathcal{N}_{\varphi}^{\kappa_{i}-1}(\varkappa, s) y_{3}(s) d s \\
\quad+\frac{\mathcal{L}_{i} w^{-1}(\varkappa)}{\Gamma\left(\kappa_{i}\right)} \int_{0}^{\varkappa} \mathcal{N}_{\varphi}^{\kappa_{i}-1}(\varkappa, s) y_{1}(\mathfrak{h}(s)) d s+\frac{\mathcal{L}_{i} w^{-1}(\varkappa)}{\Gamma\left(\kappa_{i}\right)} \int_{0}^{\varkappa} \mathcal{N}_{\varphi}^{\kappa_{i}-1}(\varkappa, s) y_{2}(\mathfrak{h}(s)) d s \\
+\frac{\mathcal{L}_{i} w^{-1}(\varkappa)}{\Gamma\left(\kappa_{i}\right)} \int_{0}^{\varkappa} \mathcal{N}_{\varphi}^{\kappa_{i}-1}(\varkappa, s) y_{3}(\mathfrak{h}(s)) d s, \quad \varkappa \in(0, b], \\
0, \quad \varkappa \in[-r, 0] .
\end{array}\right.
\end{aligned}
$$

We prove that $\mathcal{U}\left(y_{1}, y_{2}, y_{3}\right)$ is a Picard operator. For $i=1,2,3, \varkappa \in(0, b]$ and $\left(y_{1}, y_{2}, y_{3}\right)$, $\left(\widehat{y}_{1}, \widehat{y}_{2}, \widehat{y}_{3}\right) \in \Omega_{+}^{*}$, we have

$$
\begin{aligned}
&\left\|\mathcal{U}_{i}\left(y_{1}, y_{2}, y_{3}\right)-\mathcal{U}_{i}\left(\widehat{y}_{1}, \widehat{y}_{2}, \widehat{y}_{3}\right)\right\|_{E} \\
& \leq \frac{\mathcal{L}_{i} w^{-1}(\varkappa)}{\mu \Gamma\left(\kappa_{i}\right)} \int_{0}^{\varkappa} \mathcal{N}_{\varphi}^{\kappa_{i}-1}(\varkappa, s)\left|y_{1}(s)-\widehat{y}_{1}(s)\right| d s \\
& \quad+\frac{\mathcal{L}_{i} w^{-1}(\varkappa)}{\mu \Gamma\left(\kappa_{i}\right)} \int_{0}^{\varkappa} \mathcal{N}_{\varphi}^{\kappa_{i}-1}(\varkappa, s)\left|y_{2}(s)-\widehat{y}_{2}(s)\right| d s \\
& \quad+\frac{\mathcal{L}_{i} w^{-1}(\varkappa)}{\mu \Gamma\left(\kappa_{i}\right)} \int_{0}^{\varkappa} \mathcal{N}_{\varphi}^{\kappa_{i}-1}(\varkappa, s)\left|y_{3}(s)-\widehat{y}_{3}(s)\right| d s \\
& \quad+\frac{\mathcal{L}_{i} w^{-1}(\varkappa)}{\mu \Gamma\left(\kappa_{i}\right)} \int_{0}^{\varkappa} \mathcal{N}_{\varphi}^{\kappa_{i}-1}(\varkappa, s)\left|y_{1}(\mathfrak{h}(s))-\widehat{y}_{1}(\mathfrak{h}(s))\right| d s \\
& \quad+\frac{\mathcal{L}_{i} w^{-1}(\varkappa)}{\mu \Gamma\left(\kappa_{i}\right)} \int_{0}^{\varkappa} \mathcal{N}_{\varphi}^{\kappa_{i}-1}(\varkappa, s)\left|y_{2}(\mathfrak{h}(s))-\widehat{y}_{2}(\mathfrak{h}(s))\right| d s \\
& \quad+\frac{\mathcal{L}_{i} w^{-1}(\varkappa)}{\mu \Gamma\left(\kappa_{i}\right)} \int_{0}^{\varkappa} \mathcal{N}_{\varphi}^{\kappa_{i}-1}(\varkappa, s)\left|y_{3}(\mathfrak{h}(s))-\widehat{y}_{3}(\mathfrak{h}(s))\right| d s \\
& \leq \frac{2 \mathcal{L}_{i}(\varphi(b)-\varphi(0))^{\kappa_{i}}}{\mu \Gamma\left(\kappa_{i}+1\right)}\left[\left\|y_{1}-\widehat{y}_{1}\right\|_{E}+\left\|y_{2}-\widehat{y}_{2}\right\|_{E}+\left\|y_{3}-\widehat{y}_{3}\right\|_{E}\right]
\end{aligned}
$$




$$
\leq \frac{2 \mathcal{L}_{i}(\varphi(b)-\varphi(0))^{\kappa_{i}}}{\mu \Gamma\left(\kappa_{i}+1\right)}\left\|\left(y_{1}, y_{2}, y_{3}\right)-\left(\widehat{y}_{1}, \widehat{y}_{2}, \widehat{y}_{3}\right)\right\|_{E^{*}}
$$

which implies

$$
\begin{aligned}
& \left\|\mathcal{U}\left(y_{1}, y_{2}, y_{3}\right)-\mathcal{U}\left(\widehat{y}_{1}, \widehat{y}_{2}, \widehat{y}_{3}\right)\right\|_{E^{*}} \\
& =\sum_{i=1}^{3}\left\|\mathcal{U}_{i}\left(y_{1}, y_{2}, y_{3}\right)-\mathcal{U}_{i}\left(\widehat{y}_{1}, \widehat{y}_{2}, \widehat{y}_{3}\right)\right\|_{E} \\
& \quad \leq \sum_{i=1}^{3} \frac{2 \mathcal{L}_{i}(\varphi(b)-\varphi(0))^{\kappa_{i}}}{\mu \Gamma\left(\kappa_{i}+1\right)}\left\|\left(y_{1}, y_{2}, y_{3}\right)-\left(\widehat{y}_{1}, \widehat{y}_{2}, \widehat{y}_{3}\right)\right\|_{E^{*}} \\
& \quad \leq \Upsilon\left\|\left(y_{1}, y_{2}, y_{3}\right)-\left(\widehat{y}_{1}, \widehat{y}_{2}, \widehat{y}_{3}\right)\right\|_{E^{*}} .
\end{aligned}
$$

Since $\Upsilon<1$, we conclude that the operator $\mathcal{U}$ is a contraction mapping on $\Xi$ with respect to the norm

$$
\left\|\left(\varsigma_{1}, \varsigma_{2}, \varsigma_{3}\right)\right\|_{E^{*}}=\sum_{i=1}^{3}\left\|\varsigma_{i}\right\|_{E} .
$$

According to the Banach fixed point theorem, we deduce that $\mathcal{U}\left(y_{1}, y_{2}, y_{3}\right)$ is a Picard operator and $F_{\mathcal{U}}=\left(y_{1}^{*}, y_{2}^{*}, y_{3}^{*}\right)$. Now, for all $\varkappa \in(0, b]$, we have

$$
\begin{aligned}
& y_{1}^{*}(\varkappa)=\mathcal{U}_{1}\left(y_{1}^{*}, y_{2}^{*}, y_{3}^{*}\right)(\varkappa), \\
& y_{2}^{*}(\varkappa)=\mathcal{U}_{2}\left(y_{1}^{*}, y_{2}^{*}, y_{3}^{*}\right)(\varkappa),
\end{aligned}
$$

and

$$
y_{3}^{*}(\varkappa)=\mathcal{U}_{3}\left(y_{1}^{*}, y_{2}^{*}, y_{3}^{*}\right)(\varkappa)
$$

For $i=1,2,3$, we get

$$
\begin{aligned}
y_{i}^{*}(\varkappa)= & \mathcal{U}_{i}\left(y_{1}^{*}, y_{2}^{*}, y_{3}^{*}\right)(\varkappa) \\
= & \frac{\varepsilon_{i}}{\mu} E_{\kappa_{i}}(\varphi(\varkappa)-\varphi(0))^{\kappa_{i}}+\frac{\mathcal{L}_{i} w^{-1}(\varkappa)}{\Gamma\left(\kappa_{i}\right)} \int_{0}^{\varkappa} \mathcal{N}_{\varphi}^{\kappa_{i}-1}(\varkappa, s)\left[y_{1}^{*}(s)+y_{1}^{*}(\mathfrak{h}(s))\right] d s \\
& +\frac{\mathcal{L}_{i} w^{-1}(\varkappa)}{\Gamma\left(\kappa_{i}\right)} \int_{0}^{\varkappa} \mathcal{N}_{\varphi}^{\kappa_{i}-1}(\varkappa, s)\left[y_{2}^{*}(s)+y_{2}^{*}(\mathfrak{h}(s))\right] d s \\
& +\frac{\mathcal{L}_{i} w^{-1}(\varkappa)}{\Gamma\left(\kappa_{i}\right)} \int_{0}^{\varkappa} \mathcal{N}_{\varphi}^{\kappa_{i}-1}(\varkappa, s)\left[y_{3}^{*}(s)+y_{3}^{*}(\mathfrak{h}(s))\right] d s .
\end{aligned}
$$

Next, we prove that the solution $\left(y_{1}^{*}, y_{2}^{*}, y_{3}^{*}\right)$ is increasing. Let $\sigma=\max \left\{\sigma_{1}, \sigma_{2}, \sigma_{3}\right\}$, where $\sigma_{i}:=\min _{s \in(0, b]}\left[y_{i}^{*}(s)+y_{i}^{*}(\mathfrak{h}(s))\right] \in \mathbb{R}_{+}, i=1,2,3$, then for all $0 \leq \varkappa_{1}<\varkappa_{2} \leq b$, we have

$$
\begin{aligned}
y_{i}^{*}\left(\varkappa_{2}\right)-y_{i}^{*}\left(\varkappa_{1}\right) \\
=\frac{\varepsilon_{i}}{\mu}\left[E_{\kappa_{i}}\left(\varphi\left(\varkappa_{2}\right)-\varphi(0)\right)^{\kappa_{i}}-E_{\kappa_{i}}\left(\varphi\left(\varkappa_{1}\right)-\varphi(0)\right)^{\kappa_{i}}\right]
\end{aligned}
$$




$$
\begin{aligned}
& +\frac{\mathcal{L}_{i}}{\Gamma\left(\kappa_{i}\right)} \int_{0}^{\varkappa_{1}}\left[w^{-1}\left(\varkappa_{2}\right) \mathcal{N}_{\varphi}^{\kappa_{i}-1}\left(\varkappa_{2}, s\right)-w^{-1}\left(\varkappa_{1}\right) \mathcal{N}_{\varphi}^{\kappa_{i}-1}\left(\varkappa_{1}, s\right)\right]\left[y_{1}^{*}(s)+y_{1}^{*}(\mathfrak{h}(s))\right] d s \\
& +\frac{\mathcal{L}_{i} w^{-1}\left(\varkappa_{2}\right)}{\Gamma\left(\kappa_{i}\right)} \int_{\varkappa_{1}}^{\varkappa_{2}} \mathcal{N}_{\varphi}^{\kappa_{i}-1}\left(\varkappa_{2}, s\right)\left[y_{1}^{*}(s)+y_{1}^{*}(\mathfrak{h}(s))\right] d s \\
& +\frac{\mathcal{L}_{i}}{\Gamma\left(\kappa_{i}\right)} \int_{0}^{\varkappa_{1}}\left[w^{-1}\left(\varkappa_{2}\right) \mathcal{N}_{\varphi}^{\kappa_{i}-1}\left(\varkappa_{2}, s\right)-w^{-1}\left(\varkappa_{1}\right) \mathcal{N}_{\varphi}^{\kappa_{i}-1}\left(\varkappa_{1}, s\right)\right]\left[y_{2}^{*}(s)+y_{2}^{*}(\mathfrak{h}(s))\right] d s \\
& +\frac{\mathcal{L}_{i} w^{-1}\left(\varkappa_{2}\right)}{\Gamma\left(\kappa_{i}\right)} \int_{\varkappa_{1}}^{\varkappa_{2}} \mathcal{N}_{\varphi}^{\kappa_{i}-1}\left(\varkappa_{2}, s\right)\left[y_{2}^{*}(s)+y_{2}^{*}(\mathfrak{h}(s))\right] d s \\
& +\frac{\mathcal{L}_{i}}{\Gamma\left(\kappa_{i}\right)} \int_{0}^{\varkappa_{1}}\left[w_{i}^{-1}\left(\varkappa_{2}\right) \mathcal{N}_{\varphi}^{\kappa_{i}-1}\left(\varkappa_{2}, s\right)-w_{i}^{-1}\left(\varkappa_{1}\right) \mathcal{N}_{\varphi}^{\kappa_{i}-1}\left(\varkappa_{1}, s\right)\right]\left[y_{3}^{*}(s)+y_{3}^{*}(\mathfrak{h}(s))\right] d s \\
& +\frac{\mathcal{L}_{i} w^{-1}\left(\varkappa_{2}\right)}{\Gamma\left(\kappa_{i}\right)} \int_{\varkappa_{1}}^{\varkappa_{2}} \mathcal{N}_{\varphi}^{\kappa_{i}-1}\left(\varkappa_{2}, s\right)\left[y_{3}^{*}(s)+y_{3}^{*}(\mathfrak{h}(s))\right] d s \\
& +\frac{\varepsilon_{i}}{\mu}\left[E_{\kappa_{i}}\left(\varphi\left(\varkappa_{2}\right)-\varphi(0)\right)^{\kappa_{i}}-E_{\kappa_{i}}\left(\varphi\left(\varkappa_{1}\right)-\varphi(0)\right)^{\kappa_{i}}\right] \\
& +3 \mathcal{L}_{i} \sigma \frac{w^{-1}\left(\varkappa_{2}\right)-w^{-1}\left(\varkappa_{1}\right)}{\Gamma\left(\kappa_{i}+1\right)}\left(\varphi\left(\varkappa_{2}\right)-\varphi(0)\right)^{\kappa_{i}} \\
& +\frac{w^{-1}\left(\varkappa_{1}\right)}{\Gamma\left(\kappa_{i}+1\right)}\left[\left(\varphi\left(\varkappa_{2}\right)-\varphi(0)\right)^{\kappa_{i}}-\left(\varphi\left(\varkappa_{1}\right)-\varphi(0)\right)^{\left.\left.\kappa_{i}\right]\right\}}\right. \\
& >0 .
\end{aligned}
$$

Therefore $y_{i}^{*}$ is increasing for all $i=1,2,3$, and consequently $\left(y_{1}^{*}, y_{2}^{*}, y_{3}^{*}\right)$ is increasing too. Due to $\mathfrak{h}(\varkappa) \leq \varkappa$, we get $y_{i}^{*}(\mathfrak{h}(\varkappa)) \leq y_{i}^{*}(\varkappa)$ and hence

$$
\begin{aligned}
y_{i}^{*}(\varkappa)= & \mathcal{U}_{i}\left(y_{1}^{*}, y_{2}^{*}, \ldots, y_{n}^{*}\right)(\varkappa) \\
\leq & \frac{\varepsilon_{i}}{\mu} E_{\kappa_{i}}(\varphi(\varkappa)-\varphi(0))^{\kappa_{i}} \\
& +\frac{2 \mathcal{L}_{i}}{\mu \Gamma\left(\kappa_{i}\right)} \int_{0}^{\varkappa} \mathcal{N}_{\varphi}^{\kappa_{i}-1}(\varkappa, s) y_{1}^{*}(s) d s \\
& +\frac{2 \mathcal{L}_{i}}{\mu \Gamma\left(\kappa_{i}\right)} \int_{0}^{\varkappa} \mathcal{N}_{\varphi}^{\kappa_{i}-1}(\varkappa, s) y_{2}^{*}(s) d s \\
& +\frac{2 \mathcal{L}_{i}}{\mu \Gamma\left(\kappa_{i}\right)} \int_{0}^{\varkappa} \mathcal{N}_{\varphi}^{\kappa_{i}-1}(\varkappa, s) y_{3}^{*}(s) d s .
\end{aligned}
$$

In particular, if $y_{i}=\left|\widehat{\zeta}_{i}(\varkappa)-\varsigma_{i}(\varkappa)\right|,(i=1,2,3)$, from $(3.3), y_{i} \leq \mathcal{U}_{i}\left(y_{1}^{*}, y_{2}^{*}, y_{3}^{*}\right)$ by Lemma 2.8 , we obtain $y_{i} \leq y_{i}^{*}$, where $\mathcal{U}_{i}$ is an increasing Picard operator. As a result, we get

$$
\begin{aligned}
\left|\widehat{\zeta}_{i}(\varkappa)-\varsigma_{i}(\varkappa)\right| \leq & \frac{\varepsilon_{i}}{\mu} E_{\kappa_{i}}(\varphi(\varkappa)-\varphi(0))^{\kappa_{i}} \\
& +\frac{2 \mathcal{L}_{i}}{\mu \Gamma\left(\kappa_{i}\right)} \int_{0}^{\varkappa} \mathcal{N}_{\varphi}^{\kappa_{i}-1}(\varkappa, s)\left|\widehat{\zeta}_{1}(\varkappa)-\varsigma_{1}(\varkappa)\right| d s \\
& +\frac{2 \mathcal{L}_{i}}{\mu \Gamma\left(\kappa_{i}\right)} \int_{0}^{\varkappa} \mathcal{N}_{\varphi}^{\kappa_{i}-1}(\varkappa, s)\left|\widehat{\zeta}_{2}(\varkappa)-\varsigma_{2}(\varkappa)\right| d s \\
& +\frac{2 \mathcal{L}_{i}}{\mu \Gamma\left(\kappa_{i}\right)} \int_{0}^{\varkappa} \mathcal{N}_{\varphi}^{\kappa_{i}-1}(\varkappa, s)\left|\widehat{\zeta}_{3}(\varkappa)-\varsigma_{3}(\varkappa)\right| d s .
\end{aligned}
$$


It follows that

$$
\begin{aligned}
\left\|\widehat{\zeta}_{i}-\varsigma_{i}\right\|_{E} \leq & \varepsilon_{i} E_{\kappa_{i}}(\varphi(\varkappa)-\varphi(0))^{\kappa_{i}} \\
& +\frac{2 \mathcal{L}_{i}(\varphi(b)-\varphi(0))^{\kappa_{i}}}{\mu \Gamma\left(\kappa_{i}+1\right)}\left[\left\|\widehat{\zeta}_{1}-\varsigma_{1}\right\|_{E}+\left\|\widehat{\zeta}_{2}-\varsigma_{2}\right\|_{E}+\left\|\widehat{\zeta}_{3}-\varsigma_{3}\right\|_{E}\right] \\
= & \frac{\varepsilon_{i}}{\mu} E_{\kappa_{i}}(\varphi(\varkappa)-\varphi(0))^{\kappa_{i}}+\frac{2 \mathcal{L}_{i}(\varphi(b)-\varphi(0))^{\kappa_{i}}}{\mu \Gamma\left(\kappa_{i}+1\right)}\left\|\left(\widehat{\varsigma_{1}}, \widehat{\zeta}_{2}, \widehat{\zeta}_{3}\right)-\left(\varsigma_{1}, \varsigma_{2}, \varsigma_{3}\right)\right\|_{E^{*}} .
\end{aligned}
$$

Thus

$$
\begin{aligned}
& \left\|\left(\widehat{\varsigma}_{1}, \widehat{\varsigma}_{2}, \widehat{\varsigma}_{3}\right)-\left(\varsigma_{1}, \varsigma_{2}, \varsigma_{3}\right)\right\|_{E^{*}} \\
& =\sum_{i=1}^{3}\left\|\widehat{\varsigma}_{i}-\varsigma_{i}\right\|_{E} \\
& \leq \sum_{i=1}^{3} \frac{\varepsilon_{i}}{\mu} E_{\kappa_{i}}\left(\varphi(\varkappa)-\varphi(0)^{\kappa_{i}}\right. \\
& \quad+\sum_{i=1}^{3} \frac{2 \mathcal{L}_{i}(\varphi(b)-\varphi(0))^{\kappa_{i}}}{\mu \Gamma\left(\kappa_{i}+1\right)}\left\|\left(\widehat{\varsigma}_{1}, \widehat{\varsigma}_{2}, \widehat{\varsigma}_{3}\right)-\left(\varsigma_{1}, \varsigma_{2}, \varsigma_{3}\right)\right\|_{E^{*}} .
\end{aligned}
$$

As a result, we get

$$
\left\|\left(\widehat{\varsigma_{1}}, \widehat{\varsigma_{2}}, \widehat{\zeta}_{3}\right)-\left(\varsigma_{1}, \varsigma_{2}, \varsigma_{3}\right)\right\|_{E^{*}} \leq \mathcal{M} \varepsilon E_{\kappa}\left(\varphi(\varkappa)-\varphi(0)^{\kappa} .\right.
$$

Hence, equation (3.2) is UHML stable.

Next, we use the Bielecki norm $\|\cdot\|_{B}$. Let $B_{i}=E=C([-r, b], \mathbb{R})$ be the Banach spaces of continuous functions $\varsigma_{i}:[-r, b] \rightarrow \mathbb{R},(i=1,2, \ldots, n)$ with the norms

$$
\left\|S_{i}\right\|_{B_{i}}=\max _{\varkappa \in[0, b]} e^{-\delta[\varphi(\varkappa)-\varphi(0)]}\left|\varsigma_{i}(\varkappa)\right|, \quad \delta>0, i=1,2,3,
$$

where

$$
B_{i}=\left\{\varsigma_{i}:(0, b] \rightarrow \mathbb{R} ; e^{-\delta[\varphi(\varkappa)-\varphi(0)]} \varsigma_{i}(\varkappa) \in C([-r, b], \mathbb{R})\right\} .
$$

Define the product space $B=B_{1} \times B_{2} \times B_{3}$. Clearly, $B$ is a Banach space with the following Bielecki norm:

$$
\left\|\left(\varsigma_{1}, \varsigma_{2}, \varsigma_{3}\right)\right\|_{B}=\sum_{i=1}^{n}\left\|\varsigma_{i}\right\|_{B_{i}}, \quad\left(\varsigma_{1}, \varsigma_{2}, \varsigma_{3}\right) \in B .
$$

Theorem 3.3 Assume that (H1) and (H2) are satisfied. If

$$
\Upsilon_{1}=\sum_{i=1}^{3} \frac{2 \mathcal{L}_{i} e^{\delta[\varphi(b)-\varphi(0)]}}{\mu} \frac{(\varphi(b)-\varphi(0))^{\kappa_{i}}}{\Gamma\left(\kappa_{i}+1\right)}<1
$$

then system (1.1) has a unique solution $\left(\varsigma_{1}, \varsigma_{2}, \varsigma_{3}\right) \in \Xi:=E \cap \Omega \times E \cap \Omega \times E \cap \Omega$. 
Proof In order to prove the uniqueness of solution, by means of the Banach fixed point theorem, we only prove that the operator $\mathcal{G}\left(\varsigma_{1}, \varsigma_{2}, \varsigma_{3}\right)$ defined by $(2.5)$ has a fixed point in $\Xi$ with respect to Bielecki's norm. For this purpose, we divided the proof into the following steps.

Step (1): $\mathcal{G}\left(\varsigma_{1}, \varsigma_{2}, \varsigma_{3}\right)$ is continuous.

The continuity of a function $f_{i}$ implies that the operator $\mathcal{G}\left(\varsigma_{1}, \varsigma_{2}, \varsigma_{3}\right)$ is continuous too.

Step (2): $\mathcal{G}\left(\varsigma_{1}, \varsigma_{2}, \varsigma_{3}\right) \in \mathbb{P}_{\zeta^{*}}$.

Define $\mathbb{P}_{\zeta^{*}}$ is a bounded, closed, and convex set as $\mathbb{P}_{\zeta^{*}}=\left\{\left(\varsigma_{1}, \varsigma_{2}, \varsigma_{3}\right) \in B:\left\|\left(\varsigma_{1}, \varsigma_{2}, \varsigma_{3}\right)\right\|_{B} \leq\right.$ $\left.\zeta^{*}\right\}$ with

$$
\zeta^{*} \geq \frac{\Lambda_{1}}{1-\Upsilon_{1}} \quad \text { where } \Lambda_{1}:=\sum_{i=1}^{3}\left[\frac{|w(0)|}{\mu} a_{i}+\frac{2 \mathcal{L}_{i} e^{\delta[\varphi(b)-\varphi(0)]}}{\mu} \widehat{f}_{i} \frac{(\varphi(b)-\varphi(0))^{\kappa_{i}}}{\Gamma\left(\kappa_{i}+1\right)}\right]
$$

where $\widehat{f_{i}}=\sup _{s \in[0, b]}\left|w f_{i}(s, 0,0)\right|$ and $\mu=\max _{\varkappa \in[0, b]}|w(\varkappa)|$.

Case (1). For each $\left(\varsigma_{1}, \varsigma_{2}, \varsigma_{3}\right) \in \mathbb{P}_{\zeta}, i=1,2,3$, and for $\varkappa \in[-r, 0]$, we have

$$
\left\|\mathcal{G}_{i}\left(\varsigma_{1}, \varsigma_{2}, \varsigma_{3}\right)\right\|_{B_{i}}=\left\|\vartheta_{i}\right\|_{B_{i}}
$$

Hence

$$
\begin{aligned}
\left\|\mathcal{G}\left(\varsigma_{1}, \varsigma_{2}, \varsigma_{3}\right)\right\|_{B} & \leq\left\|\vartheta_{1}\right\|_{B_{i}}+\left\|\vartheta_{2}\right\|_{B_{i}}+\left\|\vartheta_{3}\right\|_{B_{i}} \\
& \leq\left\|\left(\vartheta_{1}, \vartheta_{2}, \vartheta_{3}\right)\right\|_{B} \leq \zeta^{*} .
\end{aligned}
$$

Case (2). For each $\left(\varsigma_{1}, \varsigma_{2}, \varsigma_{3}\right) \in \mathbb{P}_{\zeta^{*}}, i=1,2,3$, and for $\varkappa \in[0, b]$, we have

$$
\begin{aligned}
\left|\mathcal{G}_{i}\left(\varsigma_{1}, \varsigma_{2}, \varsigma_{3}\right)\right| & \\
\leq & \frac{|w(0)|}{|w(\varkappa)|} a_{i} \\
& +\frac{w^{-1}(\varkappa)}{\Gamma\left(\kappa_{i}\right)} \int_{0}^{\varkappa} w(s) \mathcal{N}_{\varphi}^{\kappa_{i}-1}(\varkappa, s)\left|f_{i}(s, \varsigma(s), \varsigma(\mathfrak{h}(s)))-f_{i}(s, 0,0)+f_{i}(s, 0,0)\right|(\varkappa) \\
\leq & \frac{|w(0)|}{|w(\varkappa)|} a_{i}+\frac{w^{-1}(\varkappa)}{\Gamma\left(\kappa_{i}\right)} \int_{0}^{\varkappa} \mathcal{N}_{\varphi}^{\kappa_{i}-1}(\varkappa, s) e^{\delta[\varphi(\varkappa)-\varphi(0)]} \\
& \times e^{-\delta[\varphi(\varkappa)-\varphi(0)]}\left\{\left|w(s)\left(f_{i}(s, \varsigma(s), \varsigma(\mathfrak{h}(s)))-f_{i}(s, 0,0)\right)\right|+\left|w(s) f_{i}(s, 0,0)\right|\right\} d s \\
\leq & \frac{|w(0)|}{\mu} a_{i}+\frac{2 \mathcal{L}_{i}}{\mu \Gamma\left(\kappa_{i}\right)}\left\{\left(\left\|\varsigma_{1}\right\|_{B_{i}}+\left\|\varsigma_{2}\right\|_{B_{i}}+\left\|\varsigma_{3}\right\|_{B_{i}}\right)+\widehat{f}_{i}\right\} \int_{0}^{\varkappa} \mathcal{N}_{\varphi}^{\kappa_{i}-1}(\varkappa, s) e^{\delta[\varphi(\varkappa)-\varphi(0)]} d s \\
\leq & \frac{|w(0)|}{\mu} a_{i}+\frac{2 \mathcal{L}_{i} e^{\delta[\varphi(b)-\varphi(0)]}}{\mu}\left\{\left\|\left(\varsigma_{1}, \varsigma_{2}, \varsigma_{3}\right)\right\|_{B}+\widehat{f}_{i}\right\} \frac{(\varphi(b)-\varphi(0))^{\kappa_{i}}}{\Gamma\left(\kappa_{i}+1\right)} \\
\leq & \frac{|w(0)|}{\mu} a_{i}+\frac{2 \mathcal{L}_{i} e^{\delta[\varphi(b)-\varphi(0)]}}{\mu}\left\{\zeta^{*}+\widehat{f}_{i}\right\} \frac{(\varphi(b)-\varphi(0))^{\kappa_{i}}}{\Gamma\left(\kappa_{i}+1\right)} \\
\leq & \frac{|w(0)|}{\mu} a_{i}+\frac{2 \mathcal{L}_{i} e^{\delta[\varphi(b)-\varphi(0)]}}{\mu} \frac{(\varphi(b)-\varphi(0))^{\kappa_{i}}}{\Gamma\left(\kappa_{i}+1\right)} \widehat{f}_{i} \\
& +\frac{2 \mathcal{L}_{i} e^{\delta[\varphi(b)-\varphi(0)]}}{\mu} \frac{(\varphi(b)-\varphi(0))^{\kappa_{i}}}{\Gamma\left(\kappa_{i}+1\right)} \zeta^{*},
\end{aligned}
$$


which implies

$$
\begin{aligned}
& \left\|\mathcal{G}\left(\varsigma_{1}, \varsigma_{2}, \varsigma_{3}\right)\right\|_{B} \\
& =\sum_{i=1}^{3}\left\|\mathcal{G}_{i}\left(\varsigma_{1}, \varsigma_{2}, \varsigma_{3}\right)\right\|_{B_{i}} \\
& \leq \sum_{i=1}^{3}\left[\frac{|w(0)|}{\mu} a_{i}+\frac{2 \mathcal{L}_{i} e^{\delta[\varphi(b)-\varphi(0)]}}{\mu} \widehat{f}_{i} \frac{(\varphi(b)-\varphi(0))^{\kappa_{i}}}{\Gamma\left(\kappa_{i}+1\right)}\right] \\
& \quad+\sum_{i=1}^{3} \frac{2 \mathcal{L}_{i} e^{\delta[\varphi(b)-\varphi(0)]}}{\mu} \frac{(\varphi(b)-\varphi(0))^{\kappa_{i}}}{\Gamma\left(\kappa_{i}+1\right)} \zeta^{*} \\
& <\Lambda_{1}+\zeta^{*} \Upsilon_{1} \leq \zeta^{*} .
\end{aligned}
$$

This proves that $\mathcal{G}\left(\varsigma_{1}, \varsigma_{2}, \varsigma_{3}\right) \in \mathbb{P}_{\zeta^{*}}$.

Step (3): $\mathcal{G}\left(\varsigma_{1}, \varsigma_{2}, \varsigma_{3}\right)$ is a contraction.

In this step, we need only to prove that the operator $\mathcal{G}\left(\varsigma_{1}, \varsigma_{2}, \varsigma_{3}\right)$ is a contraction mapping on $\Xi$ with respect to the Bielecki norm $B$.

Case (1): For $\left(\varsigma_{1}, \varsigma_{2}, \varsigma_{3}\right),\left(v_{1}, v_{2}, v_{3}\right) \in B$ and $\varkappa \in[-r, 0], i=1,2,3$, we have

$$
\left\|\mathcal{G}_{i}\left(\varsigma_{1}, \varsigma_{2}, \varsigma_{3}\right)-\mathcal{G}_{i}\left(v_{1}, v_{2}, v_{3}\right)\right\|_{B_{i}}=0
$$

It follows that

$$
\begin{aligned}
& \left\|\mathcal{G}\left(\varsigma_{1}, \varsigma_{2}, \varsigma_{3}\right)-\mathcal{G}\left(v_{1}, v_{2}, \nu_{3}\right)\right\|_{B} \\
& \quad=\sum_{i=1}^{n}\left\|\mathcal{G}_{i}\left(\varsigma_{1}, \varsigma_{2}, \varsigma_{3}\right)-\mathcal{G}_{i}\left(v_{1}, v_{2}, v_{3}\right)\right\|_{B_{i}} \\
& \quad<\Upsilon_{1}\left\|\left(\varsigma_{1}, \varsigma_{2}, \varsigma_{3}\right)-\left(v_{1}, v_{2}, v_{3}\right)\right\|_{B} .
\end{aligned}
$$

Case (2): For $\left(\varsigma_{1}, \varsigma_{2}, \varsigma_{3}\right),\left(v_{1}, v_{2}, v_{3}\right) \in B, \varkappa \in(0, b]$ and by $\left(\mathrm{H}_{2}\right)$, we obtain

$$
\begin{aligned}
\left\|\mathcal{G}_{i}\left(\varsigma_{1}, \varsigma_{2}, \varsigma_{3}\right)-\mathcal{G}_{i}\left(v_{1}, v_{2}, v_{3}\right)\right\|_{B_{i}} \\
\leq \frac{w^{-1}(\varkappa)}{\Gamma\left(\kappa_{i}\right)} \int_{0}^{\varkappa} w(s) \mathcal{N}_{\varphi}^{\kappa_{i}-1}(\varkappa, s)\left|\left[f_{i}(s, \varsigma(s), \varsigma(\mathfrak{h}(s)))-f_{i}(s, v(s), v(\mathfrak{h}(s)))\right]\right| \\
\leq \frac{w^{-1}(\varkappa)}{\Gamma\left(\kappa_{i}\right)} \int_{0}^{\varkappa} \mathcal{N}_{\varphi}^{\kappa_{i}-1}(\varkappa, s)\left|w(s)\left[f_{i}(s, \varsigma(s), \varsigma(\mathfrak{h}(s)))-f_{i}(s, v(s), v(\mathfrak{h}(s)))\right]\right| d s \\
\leq \frac{\mathcal{L}_{i}}{\mu \Gamma\left(\kappa_{i}\right)} \int_{0}^{\varkappa} \mathcal{N}_{\varphi}^{\kappa_{i}-1}(\varkappa, s) e^{\delta[\varphi(\varkappa)-\varphi(0)]} \\
\quad \times e^{-\delta[\varphi(\varkappa)-\varphi(0)]}\left\{\left|\varsigma_{1}(s)-v_{1}(s)\right|+\left|\varsigma_{2}(s)-v_{2}(s)\right|+\left|\varsigma_{3}(s)-v_{3}(s)\right|\right. \\
\left.\quad+\left|\varsigma_{1}(\mathfrak{h}(s))-v_{1}(\mathfrak{h}(s))\right|+\left|\varsigma_{2}(\mathfrak{h}(s))-v_{2}(\mathfrak{h}(s))\right|+\left|\varsigma_{3}(\mathfrak{h}(s))-v_{3}(\mathfrak{h}(s))\right|\right\} d s \\
\leq \frac{2 \mathcal{L}_{i} e^{\delta \varphi(b)-\varphi(0)]}}{\mu} \frac{(\varphi(b)-\varphi(0))^{\kappa_{i}}}{\Gamma\left(\kappa_{i}+1\right)}\left[\left\|\varsigma_{1}-v_{1}\right\|_{B_{i}}+\left\|\varsigma_{2}-v_{2}\right\|_{B_{i}}+\left\|\varsigma_{3}-v_{3}\right\|_{B_{i}}\right] \\
=\frac{2 \mathcal{L}_{i} e^{\delta[\varphi(b)-\varphi(0)]}}{\mu} \frac{(\varphi(b)-\varphi(0))^{\kappa_{i}}}{\Gamma\left(\kappa_{i}+1\right)}\left\|\left(\varsigma_{1}, \varsigma_{2}, \varsigma_{3}\right)-\left(v_{1}, v_{2}, v_{3}\right)\right\|_{B} .
\end{aligned}
$$


It follows that

$$
\begin{aligned}
\left\|\mathcal{G}\left(\varsigma_{1}, \varsigma_{2}, \ldots, \varsigma_{n}\right)-\mathcal{G}\left(v_{1}, v_{2}, \ldots, v_{n}\right)\right\|_{B} \\
\quad=\sum_{i=1}^{3}\left\|\mathcal{G}_{i}\left(\varsigma_{1}, \varsigma_{2}, \ldots, \varsigma_{n}\right)-\mathcal{G}_{i}\left(v_{1}, v_{2}, \ldots, v_{n}\right)\right\|_{B_{i}} \\
\quad \leq \sum_{i=1}^{3} \frac{2 \mathcal{L}_{i} e^{\delta[\varphi(b)-\varphi(0)]}}{\mu} \frac{(\varphi(b)-\varphi(0))^{\kappa_{i}}}{\Gamma\left(\kappa_{i}+1\right)}\left\|\left(\varsigma_{1}, \varsigma_{2}, \varsigma_{3}\right)-\left(v_{1}, v_{2}, v_{3}\right)\right\|_{B} \\
\leq \Upsilon_{1}\left\|\left(\varsigma_{1}, \varsigma_{2}, \varsigma_{3}\right)-\left(v_{1}, v_{2}, v_{3}\right)\right\|_{B} .
\end{aligned}
$$

Thus, the operator $\mathcal{G}\left(\varsigma_{1}, \varsigma_{2}, \varsigma_{3}\right)$ is a contraction mapping on $\Xi$ with respect to Bielecki's norm. So, by the above steps and the Banach fixed point theorem, we deduce that system (1.1) has a unique solution in $\Xi$.

The proof of UHML stability is just like in Theorem 3.1, so we omit it here.

\section{An example}

Example 4.1 -i Consider the following WFDE:

$$
\left\{\begin{array}{l}
{ }_{w}^{C} \mathcal{D}_{0^{+}}^{\kappa_{i}, \varphi} \varsigma_{i}(\varkappa)=f_{i}(\varkappa, \varsigma(\varkappa), \varsigma(\mathfrak{h}(\varkappa))), \quad \varkappa \in(0,1] \\
\varsigma_{i}(0)=a_{i}, \\
\varsigma_{i}(\varkappa)=\varphi_{i}(\varkappa), \quad \varkappa \in[-1,0] .
\end{array}\right.
$$

Set $\kappa_{i}=\frac{1}{i}, f_{i}(\cdot, \varsigma(\cdot), \varsigma(\mathfrak{h}(\cdot)))=\sum_{i=1}^{n}\left[\varphi_{i}(\cdot)-\varphi_{i}(0)+\frac{1}{10}\left(\cos \left(2 \varsigma_{i}(\cdot-1)\right)+\frac{\varsigma_{i}^{2}(\cdot)}{1+\varsigma_{i}^{2}(\cdot)}\right)\right], w_{i}(\varkappa)=e^{-\varkappa}$, $i=1,2,3, \mathfrak{h}(\cdot)=(\cdot-1)$. Thus, for all $\varsigma_{i}, \varsigma_{i}^{*} \in \mathbb{R}^{+}$and $\varkappa \in(0,1]$, we have

$$
\begin{gathered}
\left|w_{i}(\varkappa)\left[f_{i}(\varkappa, \varsigma(\varkappa), \varsigma(\mathfrak{h}(\varkappa)))-f_{i}\left(\varkappa, \varsigma^{*}(\varkappa), \varsigma^{*}(\mathfrak{h}(\varkappa))\right)\right]\right| \\
\leq \frac{1}{10}\left|\varsigma(\varkappa)-\varsigma^{*}(\varkappa)\right|+\frac{1}{10}\left|\varsigma(\mathfrak{h}(\varkappa))-\varsigma^{*}(\mathfrak{h}(\varkappa))\right| .
\end{gathered}
$$

Here $\mathcal{L}_{i}=\frac{1}{10}$ for all $i=1,2,3$. We select $\varphi(\varkappa):=e^{\varkappa}$. Then we get $\mu=\max _{\varkappa \in(0,1]}\left|e^{\varkappa}\right|=e$, and hence $\Upsilon=\sum_{i=1}^{3} \frac{2 \mathcal{L}_{i}}{\mu} \frac{(\varphi(b)-\varphi(0))^{\kappa_{i}}}{\Gamma\left(\kappa_{i}+1\right)} \simeq 0.5$. Thus all conditions in Theorem 3.1 are satisfied, and hence system (4.1) has a unique solution. Finally, we see that the inequality

$$
\mid{ }_{w}^{C} \mathcal{D}_{0^{+}}^{\kappa_{i}, \varphi} \widehat{\zeta}_{i}(\varkappa)-f_{i}\left(\varkappa, \widehat{\zeta}, \widehat{\zeta}(\mathfrak{h}(\varkappa)) \mid \leq \varepsilon_{i} E_{\kappa_{i}}(\varphi(\varkappa)-\varphi(0))^{\kappa_{i}}\right.
$$

is satisfied. Then equation (3.2) is UHML stable with

$$
\left\|\left(\widehat{\varsigma}_{1}, \widehat{\varsigma}_{2}, \widehat{\varsigma}_{3}\right)-\left(\varsigma_{1}, \varsigma_{2}, \varsigma_{3}\right)\right\|_{E^{*}} \leq \mathcal{M} \varepsilon E_{\kappa}\left(\varphi(\varkappa)-\varphi(0)^{\kappa},\right.
$$

where $\mathcal{M} \simeq 2.2>0$.

\section{Conclusion}

We have obtained the existence of unique solutions and Ulam-Hyers-Mittag-Leffler stability results for the solution of a tripled system of weighted generalized Caputo fractional derivatives investigated by Jarad et al. [1] with respect to Chebyshev and Bielecki norms 
and time delay based on the reduction of fractional differential equations to integral equations. We employed the Picard operator method and fixed point theorems to obtain our results. To the best of our observation, there is no analytical literature on studying the existence of tripled systems of fractional differential equations. This paper is the first work to study existence of a unique solution and an Ulam-Hyers-Mittag-Leffler stability result for (TSWFDEs) with respect to Chebyshev and Bielecki norms with time delay. We trust the reported results here will have a positive impact on the event of further applications in engineering and applied sciences.

\section{Acknowledgements}

All authors would like to thank their institutions for the support.

\section{Funding}

Not applicable.

Availability of data and materials

Data sharing is not applicable to this article as no datasets were generated or analysed during the current study.

\section{Competing interests}

The authors declare that they have no competing interests.

\section{Authors' contributions}

All authors equally contributed this manuscript and approved the final version.

\section{Author details}

'Department of Mathematics, Dr. Babasaheb Ambedkar Marathwada University, Aurangabad, (MS) 431001, India. 2Department of Mathematics, Hajjah University, Hajjah, Yemen. ${ }^{3}$ Department of Mathematics, Çankaya University, 06790 Etimesgut, Ankara, Turkey. ${ }^{4}$ Department of Medical Research, China Medical University, Taichung 40402, Taiwan. ${ }^{5}$ Department of Mathematics and General Sciences, Prince Sultan University, Riyadh, Saudi Arabia. ${ }^{6}$ Department of Computer Science and Information Engineering, Asia University, Taichung, Taiwan.

\section{Publisher's Note}

Springer Nature remains neutral with regard to jurisdictional claims in published maps and institutional affiliations.

Received: 29 January 2021 Accepted: 9 June 2021 Published online: 16 June 2021

\section{References}

1. Jarad, F., Abdeljawad, T., Shah, K.: On the weighted fractional operators of a function with respect to another function. Fractals 28(08), 2040011 (2020)

2. Podlubny, I.: Fractional Differential Equations. Academic Press, New York (1999)

3. Kilbas, A.A., Srivastava, H.M., Trujillo, J.J.: Theory and Applications of the Fractional Differential Equations. North-Holland Mathematics Studies, vol. 204 (2006)

4. Samko, S.G., Kilbas, A.A., Marichev, O.I.: Fractional Integrals and Derivatives. Gordon \& Breach, Yverdon (1993)

5. Atangana, A., Baleanu, D.: New fractional derivatives with non-local and non-singular kernel: theory and application to heat transfer model. Therm. Sci. 20(2), 763-769 (2016)

6. Abdeljawad, T., Baleanu, D.: On fractional derivatives with generalized Mittag-Leffler kernels. Adv. Differ. Equ. 2018, $468(2018)$

7. Almeida, R.: A Caputo fractional derivative of a function with respect to another function. Commun. Nonlinear Sci. Numer. Simul. 44, 460-481 (2017)

8. Almeida, R., Malinowska, A.B., Odzijewicz, T.: Fractional differential equations with dependence on the Caputo-Katugampola derivative. J. Comput. Nonlinear Dyn. 11(6), 061017 (2016). https://doi.org/10.1115/1.4034432

9. Atangana, A.: Fractal-fractional differentiation and integration: connecting fractal calculus and fractional calculus to predict complex system. Chaos Solitons Fractals 102, 396-406 (2017)

10. Jarad, F., Abdeljawad, T.: Generalized fractional derivatives and Laplace transform. Discrete Contin. Dyn. Syst., Ser. S 13(3), 709-722 (2020)

11. Jarad, F., Abdeljawad, T., Baleanu, D.: Caputo-type modification of the Hadamard fractional derivatives. Adv. Differ. Equ. 2012(1), 142 (2012)

12. Kilbas, A.A., Srivastava, H.M., Trujillo, J.J.: Theory and Applications of Fractional Differential Equations. Elsevier, Amsterdam (2006)

13. Katugampola, U.N.: A new approach to generalized fractional derivatives. Bull. Math. Anal. Appl. 6(4), 1-15 (2014)

14. Oliveira, D.S., de Oliveira, E.C.: Hilfer-Katugampola fractional derivatives. Comput. Appl. Math. 37(3), 3672-3690 (2018)

15. Sousa, J.V.C., de Oliveira, C.E.: On the $\psi$-Hilfer fractional derivative. Commun. Nonlinear Sci. Numer. Simul. 60, 72-91 (2018)

16. Abdeljawad, T:: Fractional operators with generalized Mittag-Leffler kernels and their differintegrals. Chaos 29 023102 (2019). https://doi.org/10.1063/1.5085726 
17. Abdeljawad, T:: Fractional difference operators with discrete generalized Mittag-Leffler kernels. Chaos Solitons Fractals 126, 315-324 (2019)

18. Abdo, M.S., Hanan, A.W., Panchal, S.K.: Ulam-Hyers-Mittag-Leffler stability for a $\psi$-Hilfer problem with fractional order and infinite delay. Results Appl. Math. 7, 100115 (2020). https://doi.org/10.1016/j.rinam.2020.100115

19. Almalahi, M.A., Abdo, M.S., Panchal, S.K.: $\psi$-Hilfer fractional functional differential equation by Picard operator method. J. Appl. Nonlinear Dyn. 9(4), 685-702 (2020)

20. Almalahi, M.A., Abdo, M.S., Panchal, S.K.: Existence and Ulam-Hyers-Mittag-Leffler stability results of $\psi$-Hilfer nonlocal Cauchy problem. Rend. Circ. Mat. Palermo (2) 70(1), 57-77 (2021)

21. Almalahi, M.A., Panchal, S.K.: On the theory of $\psi$-Hilfer nonlocal Cauchy problem. J. Sib. Fed. Univ. Math. Phys. 14(2), 159-175 (2021). https://doi.org/10.17516/1997-1397-2021-14-2-161-177

22. Al-Mayyahi, S.Y., Abdo, M.S., Redhwan, S.S., Abood, B.N.: Boundary value problems for a coupled system of Hadamard-type fractional differential equations. IAENG Int. J. Appl. Math. 51(1), 1-10 (2021)

23. Abdo, M.S., Abdeljawad, T., Ali, S.M., Shah, K., Jarad, F.: Existence of positive solutions for weighted fractional order differential equations. Chaos Solitons Fractals 141, 110341 (2020)

24. Afshari, H., Atapour, M., Karapınar, E.: A discussion on a generalized Geraghty multi-valued mappings and applications. Adv. Differ. Equ. 2020, 356 (2020). https://doi.org/10.1186/s13662-020-02819-2

25. Afshari, H., Karapı, N.E.: A discussion on the existence of positive solutions of the boundary value problems via $\psi$-Hilfer fractional derivative on b-metric spaces. Adv. Differ. Equ. 2020, 616 (2020). https://doi.org/10.1186/s13662-020-03076-z

26. Afshari, H., Baleanu, D.: Applications of some fixed point theorems for fractional differential equations with Mittag-Leffler kernel. Adv. Differ. Equ. 2020, 140 (2020). https://doi.org/10.1186/s13662-020-02592-2

27. Almalahi, M.A., Panchal, S.K., Jarad, F.: Stability results of positive solutions for a system of $\psi$-Hilfer fractional differential equations. Chaos Solitons Fractals 147, 110931 (2021)

28. Ahmad, B., Ntouyas, S.K.: Existence results for a coupled system of Caputo type sequential fractional differential equations with nonlocal integral boundary conditions. Appl. Math. Comput. 266, 615-622 (2015)

29. Almalahi, M.A., Abdo, M.S., Panchal, S.K.: Existence and Ulam-Hyers stability results of a coupled system of $\psi$-Hilfer sequential fractional differential equations. Results Appl. Math. 10, 100142 (2021)

30. Ahmad, B., Ntouyas, S.K., Alsaedi, A.: On a coupled system of fractional differential equations with coupled nonlocal and integral boundary conditions. Chaos Solitons Fractals 83, 234-241 (2016)

31. Alsulami, H.H., Ntouyas, S.K., Agarwal, R.P., Ahmad, B., Alsaedi, A.: A study of fractional-order coupled systems with a new concept of coupled non-separated boundary conditions. Bound. Value Probl. 2017, 68 (2017)

32. Berinde, V., Borcut, M.: Tripled fixed point theorems for contractive type mappings in partially ordered metric spaces. Nonlinear Anal., Theory Methods Appl. 74(15), 4889-4897 (2011)

33. Karakaya, K., Bouzara, N.-E., DoLan, K., Atalan, Y.: Existence of tripled fixed points for a class of condensing operators in Banach spaces. Sci. World J. 2014, Article ID 541862 (2014). https://doi.org/10.1155/2014/541862

34. Matar, M.M., Amra, I.A., Alzabut, J.: Existence of solutions for tripled system of fractional differential equations involving cyclic permutation boundary conditions. Bound. Value Probl. 2020, 140 (2020)

35. Wang, J., Zhang, Y.: Ulam-Hyers-Mittag-Leffler stability of fractional-order delay differential equations. Optimization $63,1181-1190(2014)$

\section{Submit your manuscript to a SpringerOpen ${ }^{\circ}$ journal and benefit from:}

- Convenient online submission

- Rigorous peer review

- Open access: articles freely available online

- High visibility within the field

- Retaining the copyright to your article

Submit your next manuscript at $\gg$ springeropen.com 